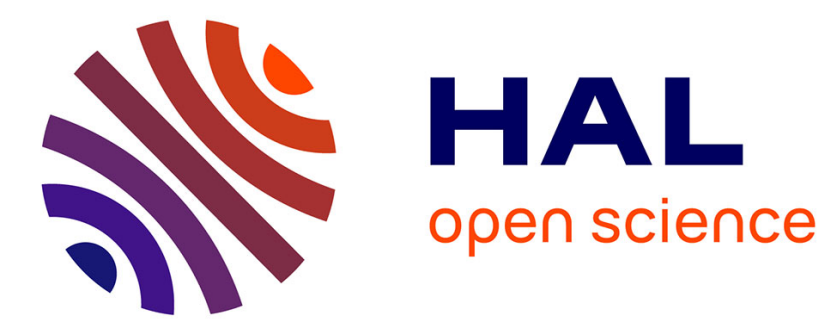

\title{
Discounting and Divergence of Opinion
}

\author{
Elyès Jouini, Jean-Michel Marin, Clotilde Napp
}

\section{To cite this version:}

Elyès Jouini, Jean-Michel Marin, Clotilde Napp. Discounting and Divergence of Opinion. Journal of Economic Theory, 2010, 145, pp.830-859. halshs-00176636v3

\section{HAL Id: halshs-00176636 \\ https://shs.hal.science/halshs-00176636v3}

Submitted on 2 Jun 2010

HAL is a multi-disciplinary open access archive for the deposit and dissemination of scientific research documents, whether they are published or not. The documents may come from teaching and research institutions in France or abroad, or from public or private research centers.
L'archive ouverte pluridisciplinaire HAL, est destinée au dépôt et à la diffusion de documents scientifiques de niveau recherche, publiés ou non, émanant des établissements d'enseignement et de recherche français ou étrangers, des laboratoires publics ou privés. 


\title{
Discounting and Divergence of Opinion*
}

\author{
Elyès Jouini ${ }^{\dagger} \quad$ Jean-Michel Marin ${ }^{\ddagger} \quad$ Clotilde Napp ${ }^{\mathbf{g}}$
}

September 16, 2008

\begin{abstract}
The objective of this paper is to adopt a general equilibrium model and determine the socially efficient discount rate when there are heterogeneous anticipations about the future of the economy as well as heterogeneous time preference rates. Among others we tackle the following questions. Is the socially efficient discount factor an arithmetic average of the individual subjectively anticipated discount factors as in the certainty equivalent approach of Weitzman $(1998,2001)$ ? As a sort of additional risk or uncertainty, can beliefs dispersion lead to lower discount rates ? Is it socially efficient, when diversity of opinion is taken into account, to reduce the discount rate per year for more distant horizons ? Does the socially efficient discount rate converge in the long run to the lowest individual subjectively anticipated discount rate as in the homogeneous beliefs setting? More generally, what is the shape of the yield curve?
\end{abstract}

\section{Introduction}

The concept of a discount rate is central to economic analysis, as it allows effects occurring at different future times to be compared by converting each future dollar amount into equivalent present dollars. The problem of the determination of a discount rate has acquired renewed relevance lately in order to analyse environmental projects or activities ${ }^{1}$ the effects of which will be spread out over hundreds of years, and the evaluation of which, through Costs and Benefits Analysis (CBA), is very sensitive to the discount rate being used. For instance, concerning global climate change, it has been argued that the strong conclusions of the Stern Review were essentially driven by the low assumed discount rate (see, e.g. Nordhaus, 2007 or Weitzman, 2007).

As underlined by e.g. Nordhaus (2007) or Weitzman (2007), there is an important distinction between the utility social discount rate and the consumption social discount rate. The former refers to a pure time preference rate that discounts utility. It reflects the level of impatience

\footnotetext{
*The financial support of the GIP ANR and of the Risk Foundation (Groupama Chair) are gratefully acknowledged by the authors. We have benefited from comments from participants at the 6 th Toulouse Conference on Environment and Resources Economics and at the FIME (EDF-Dauphine) seminar.

${ }^{\dagger}$ CEREMADE, Université Paris Dauphine, Paris, F-75775 Paris cedex 16.

${ }^{\ddagger}$ INRIA FUTURS, Projet Select, Université Paris-Sud

${ }^{\S}$ CNRS, UMR 7088, F-75016 Paris.

๑Université Paris Dauphine, DRM, F-75016 Paris and Crest

${ }^{1}$ Prominent examples include: global climate change, radioactive waste disposal, loss of biodiversity, thinning of stratospheric ozone, groundwater pollution, minerals depletion, and many others.
} 
or, for long time horizon projects, the relative weights of different people or generations. The latter is the rate used to discount future consumption. There are essentially three determinants of the level of this discount rate. The first determinant is related to a psychological "preference for the present" effect and is represented by the utility discount rate. The more impatient the individuals, the higher the value of one unit of consumption today relative to one unit of consumption tomorrow, the higher the discount rate. But there are other reasons to discount future consumption. The second determinant is related to a wealth effect while the third determinant is related to a precautionary savings effect. These two effects are directly impacted by agents' beliefs about the future of the economy. In this paper we are interested in the properties of the consumption social discount rate since our aim is to determine the value today (in present dollars) of future dollars amount in order to apply it for CBA.

A critical feature that must be taken into account is divergence of opinion about the future of the economy. Forecasting for the coming year is already a difficult task. It is natural that forecasts for the next century/millennium are subject to potentially enormous divergence. It is doubtful that agents or economists currently have a complete understanding of the determinants of long term economic evolutions. The debate on the notion of sustainable growth is an illustration of the degree of possible divergence of opinion about future of society. Some will argue that the effects of improvement in information technology have yet to be realized and the world faces a period of more rapid growth. On the contrary, those who emphasise the effects of natural resource scarcity will see lower growth rates in the future. Some even suggest a negative growth of the GNP per head in the future, due to the deterioration of the environment, population growth and decreasing return to scales.

Another critical feature that must be taken into account is the heterogeneity of utility discount rates among agents. These rates may reflect different levels of impatience. In a setting with long-lived agents that represent present and future generations, these rates may also reflect divergence of opinion about the importance granted to the welfare of future generations relative to the present. The important debate among economists (and also among philosophers) on the notion of intergenerational equity is an illustration of this possible divergence. Some will argue that intergenerational choices should be treated as intertemporal individual choices leading to weigh more present welfare. Others will argue that fundamental ethics require intergenerational neutrality and that the only ethical basis for placing less value on the welfare of future generations is the uncertainty about whether or not the world will exist and whether or not these generations will be present.

In this paper we explicitly take into account possible disagreement among agents about the future of the economy and about the treatment of future generations. As underlined by Weitzman (2001), "these and many more are fundamentally matters of judgment or opinion, on which fully informed and fully rational individuals might be expected to differ". In such a framework, our aim is to determine the socially efficient discount rate and to analyse its properties; in particular, we want to determine the analog of Ramsey Equation ${ }^{2}$ in a fairly

\footnotetext{
${ }^{2}$ In a deterministic setting, the well-known Ramsey equation gives the following expression for the discount rate$$
R=\rho+\frac{1}{\eta} g
$$ 
general heterogeneous, time-varying and stochastic ${ }^{3}$ setting. Among others, we wish to tackle the following questions. Is there general equilibrium foundation for synthesizing opinions by taking an average as in the certainty equivalent approach of Weitzman $(1998,2001)$ ? How do discount rates vary with the degree of beliefs and tastes divergence? Is it socially efficient, when there is beliefs heterogeneity, to reduce the discount rate per year for more distant horizons ? More generally, what is the shape of the yield curve?

The last questions about the shape of the yield curve are of particular interest. There is a wide agreement that discounting at a constant positive rate for long time horizons is problematic, irrespective of the particular discount rate employed. Indeed, with a constant rate, the costs and benefits accruing in the distant future appear relatively unimportant in present value terms. Hence decisions made today on this basis may expose us to catastrophic consequences in a distant future. Weitzman (1998) summarises this succinctly when he states : « To think about the distant future in terms of standard discounting is to have an uneasy intuitive feeling that something is wrong somewhere ». A recently proposed solution to this problem is to use a discount rate which declines over time. It is clear that using a declining discount rate could make an important contribution towards the goal of sustainable development. But what formal justifications exist for using a declining discount rate and what is the optimal trajectory of the decline $^{4}$ ? In a deterministic world, decreasing discount rates can arise as a result of known changes in the growth rate, changes in risk aversion, etc. Additional motivations emerge once uncertainty is considered. For example, Weitzman $(1998,2001)$ considers uncertainty on the discount rate itself. Starting from the fact that there is a huge divergence of expectations about future discount rates among economic experts, Weitzman $(1998,2001)^{5}$ introduces a probability distribution for the future discount rate and its behaviour over time. The author adopts a certainty equivalent analysis in order to determine the discount rates for varying horizons and obtains decreasing discount rates. More generally, Gollier (2002a), Dybvig et al.(1996), Weitzman $(1998,2001)$ show in different contexts that from today's perspective, the only relevant limiting scenario is the one with the lowest interest rate. In the presence of uncertain growth, Gollier (2002a, 2002b) shows that the shape of the yield curve depends upon preferences for risk and prudence, and higher order moments of the utility function. Regardless of whether it is the discount rate or the growth rate that is uncertain, the nature of the distribution of random growth is of particular importance; for instance, decreasing discount rates are obtained with Bayesian learning in Weitzman (2004), and Gollier (2007) shows that serial correlation in the growth rates leads to downward sloping yield curves when the representative agent is prudent. Decreasing discount rates also emerge from the specification of a sustainable welfare function à la Chichilnisky (1996) and Li and Löfgren (2000). Lastly, there is considerable empirical and experimental evidence to show that individuals are frequently hyperbolic discounters (see,

where $\rho$ denotes the rate of pure time preference, $g$ is the per capita growth rate of consumption and $1 / \eta$ is the elasticity of marginal utility, or equivalently the degree of relative risk aversion.

${ }^{3}$ As in standard models of the term structure of interest rates (see, e.g., Cox et al., 1985, Ingersoll and Ross, 1992, Vasicek, 1977, Cochrane, 2001).

${ }^{4}$ See Groom et al. (2005) for a survey.

${ }^{5}$ Weitzman (2001) undertakes a survey of over 2000 academic economists, and a so-called blue ribbon selection of 50, as to their opinion on the constant rate of discount to use for Cost Benefit Analysis. The responses were distributed with a gamma distribution with mean $4 \%$ and standard deviation of $3 \%$. 
e.g. Loewenstein and Thaler, 1989, Loewenstein and Prelec, 1992). In this paper, we want to examine if divergence of beliefs and heterogeneity of time preference rates can be a justification for the use of declining discount rates in a general equilibrium framework. More generally, we want to analyse if the equilibrium yield curve with beliefs and tastes heterogeneity has desirable properties.

Our theoretical framework builds upon Jouini and Napp (2007) and generalizes it to take into account heterogeneous time preference rates and infinite horizon. The agents hold heterogeneous expectations about the future of the economy. Our model encompasses disagreement about the future growth rate or about its distribution and dynamics (since we allow for stochastic growth rates) or, for example, disagreement about the possible impact of economic activity on the climate $^{6}$, or disagreement about the estimation of the possible economic damages that would be induced by climate change. In our model, the agents also differ in their rate of pure time preference. This may reflect different levels of impatience as well as different conceptions of intergenerational equity or disagreement about the probability that the world will exist at a given future date (see the Stern Report for the link between this probability and the pure time preference rate). Note that while the aim of Jouini and Napp (2007) was to provide an aggregation procedure, the aim of this paper is to analyse the properties of equilibrium discount rates. As in Gollier (2002a, 2002b), Weitzman (1998, 2001, 2007) and Nordhaus (2007), we focus on an exchange economy in order to analyse the tradeoff between current and future wealth, in particular for CBA. Nevertheless, our model can also shed some light on the analysis of the tradeoff between current production and the environmental and economic welfare of future generations; indeed, our results permit to characterise the marginal cost we are willing to invest in any technology that can reduce climate change impact in the future.

We provide the following answers to the questions above. We first obtain that the certainty equivalent approach of Weitzman $(1998,2001)$ and Reinschmidt (2002), that consists in taking the arithmetic average ${ }^{7}$ of the individually recommended discount factors is compatible with an equilibrium approach if we assume that all utility functions are logarithmic and that all the agents have the same endowment and the same time preference rate ${ }^{8}$. More generally, for logarithmic utility functions, the socially efficient discount factor is given by a weighted average of the individually recommended discount factors, the weights being determined by the individual time preference rates and the initial endowments. For more general CRRA utility functions with relative risk aversion level $1 / \eta$, the right concept of average to consider within an equilibrium approach is not the arithmetic average but an $\eta$-average. This average is, as in the logarithmic case, a weighted average. Finally, there is a bias induced by agents heterogeneity. Except in very specific settings, it is not possible to recover the socially efficient discount factor as an average of the individual subjectively anticipated ${ }^{9}$ discount factors. These

\footnotetext{
${ }^{6}$ One agent may assume that economic activity will lead to a $1^{\circ} \mathrm{C}$ excess temperature in 50 years with a $95 \%$ confidence interval of $0.5^{\circ} \mathrm{C}$, while another agent may assume that economic activity will lead to a $3^{\circ} \mathrm{C}$ excess temperature with a $95 \%$ confidence interval of $1.5^{\circ} \mathrm{C}$.

${ }^{7}$ See also Nocetti et al. (2008) for an approach leading to (possibly weighted) arithmetic averages.

${ }^{8}$ More precisely, it suffices that the product of the endowment and of the time preference rate be the same for all the agents.

${ }^{9}$ For a given agent $i$, the individual subjectively anticipated prices, discount rates and discount factors are those that would prevail if the economy was made of agent $i$ only.
} 
specific settings are first, the already mentioned logarithmic utility setting, and second, the setting with deterministic heterogeneity in pure time preference rates and no beliefs dispersion (which includes the setting with rational agents and deterministic time preference rates of Gollier and Zeckhauser, 2005, or Lengwiller, 2005). In a general setting, there is an aggregation bias and we show that it can be quite significant. The bias can be towards higher or lower discount rates depending on the relative position of $\eta$ with respect to 1 . This result is consistent with the interpretation of beliefs and time preference heterogeneity as an additional source of risk or uncertainty in the future, leading agents to value more or less future consumption (with respect to present consumption) depending on the relative level of prudence and risk aversion.

We examine the impact of beliefs and time preference heterogeneity on the expression of the discount rate as well as on the relationship between the discount rate and the time horizon (the possible shapes of the yield curve). Increased beliefs dispersion leads to a decrease of discount rates when $\eta$ is greater than one. We show that aggregate pessimism as well as aggregate patience reduce the socially efficient discount rate. Since these aggregate levels are given by stochastic, time-varying (risk-tolerance) weighted averages of the individual levels of pessimism and patience, possible correlation effects are induced. This leads in the medium term to a rich class of possible shapes for the yield curve. In particular, our model can fit observed behavior of the yield curve in financial markets where the "long term rate" (30 years) is usually higher than the short term rate. More precisely, our model is compatible with yield curves that are increasing in the short term and in the medium term (that corresponds to the financial markets long term) and that are decreasing in the long term.

We show that the yield curve is always decreasing in the (very) long run. Indeed, the bias due to beliefs dispersion vanishes in the long run and the asymptotic discount rate is given by the lowest individually anticipated asymptotic discount rate among all agents ${ }^{10}$. Note that this lowest individually anticipated asymptotic discount rate does not necessarily correspond to the rate of the most patient agent as in the homogeneous beliefs setting. For example, in the case of homogeneous time preference rates, the asymptotic discount rate is given by the discount rate of the most pessimistic agent which would consist to focus on the worst case scenario among the agents (if each agent is associated to some scenarios). More generally, both the distributions of time preference rates and of pessimism are necessary to determine the asymptotic discount rate. In the setting of a CBA, this leads to discount long term costs and benefits at the lowest anticipated rate inducing a bias towards the optimal policy of the agent who values the most future consumption in the long term. In fact, the agent who values the most future consumption (either because she is very pessimistic about the future or because she is very patient or any combination of these two possibilities) makes the market for long term bonds and therefore imposes her price. This provides us with a guideline for long term CBA. This is especially useful since, while the observed risk free rate provides a useful tool for CBA in the short term, financial markets are not very helpful when benefits and costs of the set of current potential actions are expected to last in the medium and/or in the long run ${ }^{11}$.

\footnotetext{
${ }^{10}$ Note that we retrieve at the consumption discount rate level results that are already known at the utility discount rate level. This transfer of properties from utility to consumption discount rates is immediate when agents share the same beliefs.

${ }^{11}$ For example, greenhouse gas that one emits today yields very long term costs like global warming. Liquid
} 
The paper is organized as follows. Section 2 presents the theoretical framework. Section 3 deals with socially efficient discount factors and discount rates and in particular, analyses their link with their individual subjectively anticipated counterparts. Section 4 is devoted to long term considerations. In Section 5, we analyse more in detail the shape of the yield curve in specific settings. The conclusion summarises the main contributions of the paper. Proofs of aggregation results and other extensions of Jouini-Napp (2007) are in Appendix A. Appendix $\mathrm{B}$ consists of the proofs of all other results.

\section{The theoretical framework}

We consider a continuous-time Arrow-Debreu economy with an infinite horizon, in which risk averse agents try to maximize the expected utility of future consumption. A filtered probability space $\left(\Omega, F,\left(F_{t}\right), P\right)$ is given. Each agent indexed by $i=1, \ldots N$, has a current endowment at time $t$ denoted by $e_{t}^{*^{i}}$ and a Von Neuman-Morgenstern utility function for future consumption of the form $E^{Q^{i}}\left[\int_{0}^{\infty} \exp ^{-\int_{0}^{t} \rho^{i}(s, \omega) d s} u\left(c_{t}(\omega)\right) d t\right]$, which means that agents may differ in their subjective beliefs (represented by the probability measure $Q^{i}$ ), in their pure time preference rate process $\rho^{i}$, and in their endowment process $e^{*^{i}}$. The utility function $u$ can represent the individual's utility function but may also incorporate the preferences of his descendants.

As in Jouini-Napp (2007), agents have different expectations about the future of the economy. In a purely financial framework, such beliefs heterogeneity might result, for instance, from different subjective probabilities of occurrence of a boom or of a krach. In a macroeconomic setting, beliefs heterogeneity might result from heterogeneous growth rate forecasts. More generally, beliefs heterogeneity might also result from differences of opinion about the likelihood of a catastrophe, about the probability distribution of the impact of human activity on the climate or on the possible damages resulting from climate change. In our model, agents may also disagree about the pure time preference rate. This may reflect different levels of preference for the present. With long-lived agents that represent present and future generations, this reflects different conceptions of intergenerational equity.

For tractability reasons and in order to focus on the impact of beliefs and time preference rate heterogeneity, we restrict our analysis to homogeneous utility functions of the power type, i.e. we suppose that agents share the same CRRA utility function ${ }^{12}$ for consumption, of the form $u^{\prime}(x)=x^{-1 / \eta}$. As far as beliefs heterogeneity is concerned, the unique assumption we essentially make is the equivalence of the probability measures $Q^{i}$. In other words, we assume that the agents have the same set of possible events (i.e. events with a positive subjective probability ${ }^{13}$ ). Letting $\left(M^{i}\right)$ denote the positive density process of $Q^{i}$ with respect to $P$ and letting $D_{t}^{i} \equiv$ $\exp ^{-\int_{0}^{t} \rho^{i}(s, \omega) d s}$ denote the individual pure time preference discount factor at time $t$, the utility function of agent $i$ can equivalently be written in the form $E\left[\int_{0}^{\infty} M_{t}^{i}(\omega) D_{t}^{i}(\omega) u\left(c_{t}(\omega)\right) d t\right]$.

We let $e^{*} \equiv \sum_{i=1}^{N} e^{*^{i}}$ denote the aggregate endowment process. We make the assumption

financial instruments with such large durations do not exist. For the sake of comparison, US treasury bonds have time horizons that do not exceed 30 years.

${ }^{12}$ Our approach can be extended to the case with HARA utility functions of the form $u^{\prime}(x)=(\theta+x)^{-\frac{1}{\eta}}$.

${ }^{13}$ Note that this is a natural assumption for the existence of an equilibrium. Otherwise some agents will consider as possible some events that are considered as impossible by others and optimal demand in the associated ArrowDebreu asset will be (positively or negatively) infinite. 
that $e^{*}$ and $M^{i}$ satisfy the following stochastic differential equations

$$
\left\{\begin{array}{c}
d e_{t}^{*}=\mu_{t} e_{t}^{*} d t+\sigma_{t} e_{t}^{*} d W_{t} \quad e_{0}^{*}=1 \\
d M_{t}^{i}=\delta_{t}^{i} M_{t}^{i} d W_{t}
\end{array}\right.
$$

where $W$ denotes a standard unidimensional $\left(\left(F_{t}\right), P\right)$-Brownian motion and where $\mu_{t}, \sigma_{t}$ and $\delta_{t}^{i}$ stand respectively for $\mu(t, \omega), \sigma(t, \omega)$ and $\delta^{i}(t, \omega)$, which means that they might depend upon time and states of the world. There is no Markovian assumption; the coefficients $\mu, \sigma$ and $\delta^{i}$ for $i=1, \ldots, N$ may depend on the entire past history of the economy ${ }^{14}$.

Let us recall the following notation. For a given process $\left(\delta_{t}\right)$, the Doléans-Dade exponential $\mathcal{E}_{t}(\delta)$ denotes the quantity $\exp \left(\int_{0}^{t} \delta_{s} d W_{s}-\frac{1}{2} \int_{0}^{t} \delta_{s}^{2} d s\right)$. With this notation we have, for all $i$, $M_{t}^{i}=\mathcal{E}_{t}\left(\delta^{i}\right)$.

According to Girsanov Theorem, for agent $i$, aggregate endowment is an Itô process with diffusion parameter $\sigma(t, \omega)$ and with subjective drift parameter $\mu^{i}(t, \omega) \equiv \mu(t, \omega)+\sigma(t, \omega) \delta^{i}(t, \omega)$, which means that in our model, agents differ in their expected instantaneous growth rate of aggregate endowment and agree about its volatility ${ }^{15}$. Note that even if the agents agree on the instantaneous level of risk $\sigma(t, \omega)$, the fact that $\mu^{i}(t,$.$) is stochastic permits disagreement$ among agents about the level of risk. For instance, agents for which the stochastic process $\mu^{i}$ exhibits positive (resp. negative) serial dependence will weigh more (resp. less) extreme events (see Gollier, 2007). Even in the extreme case where there is no time dependence, where $\sigma$ is constant and where $\mu^{i}$ is a discrete random variable (independent of $t$ and of $W$ ), the variance of the logarithmic return between date 0 and date $T$ is equal to $\sigma^{2} T+\operatorname{Var}\left(\mu^{i}\right) T^{2}$ allowing for divergence of opinion among agents about the level of risk.

Note that the process $\rho^{i}$ as well as $\delta^{i}$ are $\left(F_{t}\right)$ adapted stochastic processes, which may be updated continuously according to the available information. In particular, the individual beliefs $\delta^{i}$ we consider might result from Bayesian updating as in e.g. Detemple and Murthy (1994) and Zapatero (1998) or from adaptative learning as in Brock and Durlauf (2001). For instance, if the states of the world are associated to different possible levels of climate change (different levels of excess temperature), $E\left[\int_{t}^{T} \mu^{i}(s, \omega) d s \mid F_{t}\right]$ will represent the average logarithmic return between dates $t$ and $T$ that is anticipated by agent $i$ at date $t$ based on date $t$ scientific knowledge about climate change and its possible impact on the economic growth.

In our general framework, an Arrow-Debreu equilibrium is defined, as usual, by a positive price process $q^{*}$ and a family of optimal consumption plans $\left(y^{*^{i}}\right)_{i=1, \ldots, N}$ such that markets clear, i.e.

$$
\left\{\begin{array}{c}
y^{*^{i}}=y^{i}\left(q^{*}, M^{i}, D^{i}, e^{*^{i}}\right) \\
\sum_{i=1}^{N} y^{*^{i}}=e^{*}
\end{array}\right.
$$

where $y^{i}(q, M, D, e)=\arg \max _{E\left[\int_{0}^{\infty} q_{t}\left(y_{t}^{i}-e_{t}\right) d t\right] \leq 0} E\left[\int_{0}^{\infty} M_{t} D_{t} u\left(c_{t}\right) d t\right]$.

\footnotetext{
${ }^{14}$ We only assume that for all $T, \int_{0}^{T}\left|\mu_{t}\right| d t<\infty, \int_{0}^{T}\left|\sigma_{t}^{2}\right| d t<\infty$, and $\int_{0}^{T}\left|\left(\delta_{t}^{i}\right)^{2}\right| d t<\infty$, almost surely.

${ }^{15}$ More precisely, letting $W_{t}^{i} \equiv W_{t}-\int_{0}^{t} \delta^{i} d s$, we obtain through Girsanov Theorem that $W^{i}$ is a Brownian motion under $Q^{i}$ and $d e_{t}^{*}=\left(\mu_{t}+\sigma_{t} \delta^{i}\right) e_{t}^{*} d t+\sigma_{t} e_{t}^{*} d W_{t}^{i}$.
} 


\section{Discount factors and discount rates}

As in Jouini-Napp (2007), we start from an Arrow-Debreu equilibrium $\left(q^{*},\left(y^{*^{i}}\right)_{i=1, \ldots, N}\right)$. Note that since our utility functions satisfy Inada conditions, all equilibria are interior, hence there exist positive Lagrange multipliers $\left(\lambda_{i}\right)_{i=1, \ldots, N}$ such that for all $i$, the equality $M_{t}^{i} D_{t}^{i} u^{\prime}\left(y_{t}^{*^{i}}\right)=$ $\lambda_{i} q_{t}^{*}$ holds for all $t$. We let $\gamma_{i} \equiv \frac{\left(1 / \lambda_{i}\right)^{\eta}}{\sum_{j=1}^{N}\left(1 / \lambda_{j}\right)^{\eta}}$.

We fix a date $t>0$, at which a cost or benefit is incurred. The socially efficient discount factor $A^{t}$ between date 0 and date $t$ is the price of a zero coupon bond maturing at time $t$ and is given by $A^{t} \equiv E\left[q_{t}^{*}\right]$. The (average) socially efficient discount rate $R^{t}$ is defined as $R^{t} \equiv$ $-\frac{1}{t} \log A^{t}$. Analogously, the discount factor $A^{t, i}$ and the discount rate $R^{t, i}$ that would prevail if the economy was made of agent $i$ only are given by $R^{t, i} \equiv-\frac{1}{t} \log A^{t, i} \equiv-\frac{1}{t} \log E\left[M_{t}^{i} D_{t}^{i} u^{\prime}\left(e_{t}^{*}\right)\right]$. These are the discount factor and discount rate that agent $i$ "anticipates".

The aim of this section is twofold. First, to analyse the link between the socially efficient discount rate $R^{t}$ and the individual subjectively anticipated discount rates $R^{t, i}$. Second, to analyse the expression of the socially efficient discount rate $R^{t}$ and, in particular, how it departs from the standard setting.

We first consider to what extent individual subjectively anticipated socially efficient discount factors can be averaged into a consensus discount factor. Our question can be rephrased as follows: can the socially efficient discount factor $A^{t}$ be represented as an average of the individual $A^{t, i} ?$

Proposition 3.1. 1. If there is no beliefs heterogeneity and if time-preference rates are deterministic, i.e., if $\delta^{i}(s, \omega) \equiv \delta(s, \omega)$ and $\rho^{i}(s, \omega) \equiv \rho^{i}(s)$, then the socially efficient discount factor is an $\eta$-average of the individual subjectively anticipated socially efficient discount factors, more precisely

$$
A^{t}=\left[\sum_{i=1}^{N} \gamma_{i}\left(A^{t, i}\right)^{\eta}\right]^{1 / \eta} .
$$

2. In the general setting,

- If $\eta=1$, then $A^{t}=\sum_{i=1}^{N} \gamma_{i}\left(A^{t, i}\right)$.

- Otherwise, we have

$$
A^{t} \leq\left[\sum_{i=1}^{N} \gamma_{i}\left(A^{t, i}\right)^{\eta}\right]^{1 / \eta} \text { for } \eta<1
$$

and

$$
A^{t} \geq\left[\sum_{i=1}^{N} \gamma_{i}\left(A^{t, i}\right)^{\eta}\right]^{1 / \eta} \text { for } \eta>1
$$

with equality holding only when the divergence in individual characteristics $N^{i} \equiv$ $M^{i} D^{i}$ is deterministic, i.e. if $N^{i} / N^{j}$ is deterministic for all $(i, j)$.

Proposition 3.1 means first that the right concept of average to consider for discount factors (in the case of power utility functions) is an $\eta$-average, which is an arithmetic average only in the 
case of logarithmic utility functions. Moreover, this $\eta$-average is a weighted average, the weights being given by the parameters $\gamma_{i}$. These weights are deterministic and they will be analysed more in detail in Section 5. Finally, except in very specific settings, it is not possible to recover the socially efficient discount factor as an average of the individual subjectively anticipated discount factors. These specific settings are first, the setting with deterministic heterogeneity in pure time preference rates and no beliefs dispersion ${ }^{16}$ (which includes the setting with rational agents and deterministic time preference rates of Gollier and Zeckhauser, 2005), and second, the setting with logarithmic utility functions. In a general setting, there is an aggregation bias. The price at date 0 of a zero-coupon bond maturing at date $t$ is lower (resp. higher) than the (weighted $\eta-$ ) average of the subjectively anticipated prices for $\eta<1$ (resp. $\eta>1$ ). This means that with beliefs dispersion and/or stochastic time preference heterogeneity, individuals value less (resp. more) one unit of consumption at date $t$ when $\eta<1$ (resp. $\eta>1$ ).

As far as the magnitude of these biases are concerned, we shall see in Section 5 that the difference between the socially efficient discount rate and the rate associated to the $\eta$-average of the individual subjectively anticipated discount rates can be significant.

Let us elaborate on why these biases (with respect to the $\eta$-average) are in opposite directions depending on the position of $\eta$ with respect to 1 . The interpretation of $\eta$ as a degree of relative risk tolerance is not enlightening for our purpose. It seems more meaningful to observe that the condition $\eta \geq 1$ is equivalent, in our setting, to the condition that prudence is larger than twice absolute risk aversion. Indeed, this last condition ${ }^{17}$ appears as crucial in intertemporal choices analysis; Gollier and Kimball (1996) show that, in a standard portfolio problem, the opportunity to invest in a risky asset raises (resp. reduces) the aggregate saving if and only if absolute prudence is larger (resp. smaller) than twice absolute risk aversion. Moreover, Gollier (2000) studies the problem of the optimal use of a good whose consumption can produce damages in the future and shows that scientific progress providing information on the distribution of the intensity of damages induces earlier prevention effort only if prudence is larger than twice risk aversion. Hence, a possible interpretation of the central role of $\eta=1$ is the following. Interpret beliefs heterogeneity and time preference heterogeneity in a stochastic setting as additional risk or as less information or more uncertainty about the future. According to Gollier and Kimball (1996) or Gollier (2000), this should lead agents to value more future consumption in the case $\eta>1$ and less future consumption in the case $\eta<1$, which is essentially the result of Proposition 3.1.

Notice that Proposition 3.1 implies that the approach that consists in considering an arithmetic average of the individual subjectively anticipated discount rates is compatible with a general equilibrium approach as far as agents are endowed with logarithmic utility functions.

We now analyse more precisely the expression of the socially efficient discount rate and, in particular, we compare it with the standard setting. For this purpose, let us first recall some results about the risk free rate. In the standard setting with rational beliefs and homogeneous

\footnotetext{
${ }^{16}$ Note that no beliefs dispersion does not mean that individuals are rational; they can all share the same subjective belief. Analogously, all time preference rates $\rho^{i}$ need not be deterministic but they need to be written in the form $\rho^{i}(t, \omega)=\rho(t, \omega) a^{i}(t)$ where $\rho$ is a common term and where $a^{i}$ is a deterministic process.

${ }^{17}$ This condition has been thoroughly studied by Gollier (2000) and has appeared in different contexts (Drèze and Modigliani, 1972, Caroll and Kimball, 1996, Sinclair-Desgagné and Gabel, 1997, Dionne and Fombaron, 1996).
} 
time preference rate $\rho$, the risk free rate is given by

$$
r^{f}(s t d d)=\rho+\frac{\mu}{\eta}-\frac{1}{2} \frac{1}{\eta}\left(1+\frac{1}{\eta}\right) \sigma^{2}
$$

where all parameters $\rho, \mu, \sigma$ hence $r^{f}$ may depend upon $t$ and $\omega$. This is an extension of Ramsey Equation to a stochastic setting, which illustrates the patience effect, the wealth effect as well as the precautionary savings effect on the risk free rate. In our setting with heterogeneous time preference rates and beliefs, we obtain (see Proposition A-2 in Appendix A) that the risk free rate is given by

$$
r^{f}=\rho_{D}+\frac{\mu+\delta_{M} \sigma}{\eta}-\frac{1}{2} \frac{1}{\eta}\left(1+\frac{1}{\eta}\right) \sigma^{2}+\rho_{B}
$$

with $\rho_{D} \equiv \sum_{i=1}^{N} \tau_{i} \rho^{i}, \delta_{M} \equiv \sum_{i=1}^{N} \tau_{i} \delta^{i}$, and $\rho_{B} \equiv \frac{1}{2}(1-\eta) \operatorname{Var}^{\tau}(\delta)$ where $\tau_{i}=\frac{y^{*^{i}}}{e^{*}}$ represents agent $i$ risk tolerance and where $\operatorname{Var}^{\tau}(\delta)$ represents the variance of the $\delta^{i}$ s across the agents when agent $i$ is endowed with a weight $\tau_{i}$. In other words, the homogeneous time preference rate $\rho$ of the Ramsey formula is replaced by the average time preference rate $\rho_{D}$ and the objective growth rate $\mu$ is replaced by the average subjective growth rate $\mu+\delta_{M} \sigma$. Furthermore, there is an additional term $\rho_{B}$ that is directly related to beliefs' dispersion and whose impact is towards an increase or a decrease of the risk free rate depending on the position of $\eta$ with respect to 1. This is a straightforward generalization of Jouini-Napp (2007) that takes into account heterogeneous time preference rates. Note that "the precautionary savings term" is the same in Equations (3.1) and (3.2) since, as previously underlined, in our model all agents necessarily agree on the volatility level $\sigma$ (as a consequence of the equivalence of the subjective probability measures $Q^{i}$ ). Notice also that even when all parameters $\rho^{i}, \delta^{i}, \mu$ and $\sigma$ are constants, the risk tolerance weighted averages $\rho_{D}$ and $\delta_{M}$ as well as the variance term $\rho_{B}$ are time-varying and stochastic, hence the risk free rate is also time-varying and stochastic (which is not the case in the standard setting).

Comparing Equations (3.1) and (3.2), it is easy to see that there are essentially three possible ways through which beliefs and time preference rates heterogeneity may lead to lower risk free rates; first, a negative correlation between impatience and risk tolerance or a low level of impatience, second, a positive correlation between pessimism and risk tolerance or a high level of pessimism, third, a negative beliefs dispersion effect that corresponds to the case $\eta>1$.

The following proposition enables us to show that analogous results are obtained for the socially efficient discount rate. We adopt the same notations as in Equation (3.2).

Proposition 3.2. The socially efficient discount rate $R^{t}$ is given by

$$
\begin{aligned}
R^{t} & =-\frac{1}{t} \log E\left[D_{t} M_{t} B_{t} u^{\prime}\left(e_{t}^{*}\right)\right] \\
& =-\frac{1}{t} \log E^{\bar{Q}_{t}}\left[\exp -\int_{0}^{t} r_{s}^{f} d s\right]
\end{aligned}
$$

with $B_{t}=\exp \left(-\int_{0}^{t} \rho_{B}(s) d s\right), D_{t}=\exp \left(-\int_{0}^{t} \rho_{D}(s) d s\right), M_{t}=\mathcal{E}_{t}\left(\delta_{M}\right)$ and $\frac{d \bar{Q}_{t}}{d P}=\mathcal{E}_{t}\left(\delta_{M}-\frac{\sigma}{\eta}\right)$

Notice first through Equation (3.4) that when $r^{f}$ is deterministic (or when $r^{f}$ and $\frac{d \bar{Q}_{t}}{d P}$ are 
independent), all that we have just said about the impact of beliefs and time preference rates heterogeneity on $r^{f}$ is true of $R^{t}$. In particular, aggregate patience, aggregate pessimism, as well as increased beliefs dispersion when $\eta>1$ induce a socially efficient discount rate that is lower than in the standard setting.

More generally, the comparison of Equation (3.3) with the expression of the socially efficient discount rate in the standard setting, which is given by $R^{t}(s t d d)=-\frac{1}{t} \log E\left[\exp (-\rho t) u^{\prime}\left(e_{t}^{*}\right)\right]$, permits to exhibit three determinants of the impact of beliefs and time preference rates heterogeneity on the discount rate: first, the consensus time preference factor $D$, second, the belief (density) $M$, and third, the aggregation bias $B$. The analysis of the effect of these three factors on the discount rate (between date 0 and $t$ ) is less straightforward than for the (instantaneaous) risk free rate at a given date $t$. However, the main conclusions remain valid. Indeed, excess patience at the aggregate level, in the form of an average time preference rate $\int_{0}^{t} \rho_{D}(s) d s$ that is lower than the standard time preference rate $\int_{0}^{t} \rho(s) d s$ leads to a higher $D$ hence, ceteris paribus, to a lower discount rate $R^{t}$. Beliefs dispersion for $\eta \geq 1$ leads to a nonpositive parameter $\rho_{B}$ and to a factor $B$ that is greater than 1 , hence, ceteris paribus, to a discount rate that is lower than in the standard setting. Analogously, increased beliefs dispersion in the form of a higher $\operatorname{Var}^{\tau}(\delta)$ leads to an increase in $B$ for $\eta \geq 1$ hence, ceteris paribus, to a decrease in the discount rate. As far as aggregate pessimism is concerned, intuitively, a pessimistic belief increases the expected value of a decreasing function of the total endowment $e^{*}$, hence should lead to a lower discount rate $R$. The case with deterministic parameters $\mu_{t}$ and $\sigma_{t}$ illustrates this intuition. Indeed, it is then easy to obtain (see Appendix A, Proposition A-3) that, if the consensus belief is neutral or pessimistic, i.e., when $\delta_{M} \leq 0$, then

$$
-\frac{1}{t} \log E\left[M_{t} u^{\prime}\left(e_{t}^{*}\right)\right] \leq-\frac{1}{t} \log E\left[u^{\prime}\left(e_{t}^{*}\right)\right],
$$

hence the effect of pessimism only is toward a lower discount rate. In fact, we obtain that the impact of beliefs heterogeneity is towards a lower (resp. higher) socially efficient discount rate if the consensus belief is neutral or pessimistic $\left(\delta_{M} \leq 0\right)$ and when $\eta \geq 1$ (resp. the consensus belief is neutral or optimistic, i.e. $\delta_{M} \geq 0$, and when $\eta \leq 1$ ). This result is in the spirit of the findings of Dumas et al. (2008) that obtains, in a specific sentiment framework, that "whenever risk aversion is [an integer] greater than 1, an increase in the variance of sentiment reduces the expected values of all the future stochastic discount factors."

\section{Long term considerations}

We now turn to (very) long term considerations. In particular, is it socially efficient, when diversity of opinion is taken into account, to reduce the discount rate per year for far distant horizons? We obtain the following result.

Proposition 4.1. We suppose that for all $i$, the individual asymptotic discount rate $R^{\infty, i} \equiv$ $\lim _{t \rightarrow \infty} R^{t, i}$ exists $^{18}$. The asymptotic socially efficient discount rate exists and is given by the lowest

\footnotetext{
${ }^{18}$ These limits can be replaced by limits along sequences, i.e. $R^{\infty, i}=\lim _{n \rightarrow \infty} R^{t_{n}, i}$ for some sequence $t_{n}$ such that $\lim t_{n}=\infty$. In this case, the asymptotic socially efficient discount rate would be defined along the same sequences.
} 
subjectively anticipated discount rate i.e.

$$
R^{\infty} \equiv \lim _{t \rightarrow \infty} R^{t}=\inf \left\{R^{\infty, i}, i=1, \ldots, N\right\}
$$

Let us remark that in a model where each individual corresponds to an expert who consults a subjective model in order to recommend discount rates, the existence of $R^{\infty, i}$ for all $i$, means that each expert is able to propose an asymptotic discount rate.

Proposition 4.1 shows first that the bias, due to beliefs heterogeneity, that we have exhibited in the previous section vanishes in the long run. The socially efficient discount rate behaves asymptotically as the discount rate associated to an average of the individual subjectively anticipated discount factors. Indeed, under the conditions of the proposition, the socially efficient discount rate $R^{t}$ converges to the lowest individual subjectively anticipated discount rate as does the rate associated to any of the considered averages of the anticipated discount factors. The fact that the dispersion term vanishes in the long term may seem counterintuitive according to Equations (3.2) and (3.3). Indeed, the bias between $R$ and an average of the $R^{i} \mathrm{~s}$ is represented (up to a constant) by the variance of the $\delta^{i}$ s and it is not clear whether or not this variance term is negligible in the long run. In particular, the unweighted variance of the $\delta^{i} \mathrm{~s}$ is exogeneously given in our model and does not necessarily converge to zero. The fact that the weighted variance vanishes is then directly related to the dynamics of the stochastic weights $\tau_{i}$. Furthermore, we emphasise that the bias vanishes only asymptotically, and we shall see in the next section that we may have to consider very far horizons (hundreds of years) before observing this asymptotic behavior.

Proposition 4.1 proves foremost that, from today's perspective, among the possible subjectively anticipated asymptotic behaviors $R^{\infty, i}$, the only relevant asymptotic behavior is the one with the lowest discount rate. In other words, in a setting with heterogeneous agents, only the agent with the lowest anticipated discount rate matters in the long run. In fact, the agent who values the most future consumption (either because she is very pessimistic about the future or because she is very patient or any combination of these two possibilities) makes the market for long term bonds and therefore imposes her price. Mathematically, from today's perspectives, all the other agents become negligible in the long run, because their "weight" has been reduced by the power of compound discounting at a higher expected discount rate. Asymptotically, the value of the socially efficient discount factor is then given by the discount factor that would prevail in an economy made of the agent with the lowest rate only. In the case of homogeneous beliefs and heterogeneous time preference rates, this implies that the asymptotic discount rate is given by the rate associated with the lowest rate of impatience. In particular, the result of Gollier-Zeckhauser (2005) on the asymptotic discount rate with heterogeneous time preference rates $^{19}$ remains valid in a stochastic setting. In the case with homogeneous time preference rates and heterogeneous beliefs, Proposition 4.1 implies that the asymptotic socially efficient discount rate is given by the rate of the most pessimistic agent. More generally, both the distributions of time preference rates and of pessimism are necessary to determine the asymptotic discount rate (which is the lowest anticipated discount rate).

\footnotetext{
${ }^{19}$ See also Blanchard and Fischer (1989) and Li and Löfgren (2000).
} 
In the setting of a CBA, this leads to discount long term costs and benefits at the lowest anticipated rate inducing a bias towards the optimal policy of the agent who values the most future consumption in the long term. How does Proposition 4.1 relate to the previous results of, e.g., Dybvig et al.(1996), Gollier (2002b), Weitzman (1998, 2001), who show - in a standard stochastic setting with a representative agent with rational beliefs and a given time preference rate parameter - that the long term discount rate is associated with the scenario with the lowest possible rate (worst case scenario). Proposition 4.1 brings about an additional element in favor of the choice of low discount rates in the long run. Indeed, the result about the worst scenario implies that for each agent denoted by $i$, the asymptotic rate $R^{\infty, i}$ corresponds to his worst scenario (over possible states of the world). Proposition 4.1 implies then that the asymptotic behavior of the socially efficient discount rate is given by the lowest (among agents) of the lowest possible rate (among possible scenarios or states of the world). As an illustration, consider the simple case in which the instantaneous rate of growth of aggregate endowment denoted by $\mu$ is a discrete random variable, independent of $t$ and of $W$, taking values $\mu_{1}, \ldots, \mu_{J}$ with probability $p_{1}, \ldots, p_{J}$ and suppose that $\sigma, \delta^{i}, \rho^{i}$ are constants. We easily get that for all $i=1, \ldots N$, the asymptotic subjectively anticipated discount rate is given by the rate associated to the worst possible scenario, i.e., $R^{\infty, i}=\rho^{i}+\frac{1}{\eta}\left(\inf _{j=1, \ldots, J} \mu_{j}+\sigma \delta^{i}\right)-\frac{1}{2} \frac{1}{\eta}\left(1+\frac{1}{\eta}\right) \sigma^{2}$. According to Proposition 4.1, the socially efficient discount rate is then given by the lowest possible rate among the agents, i.e. $R^{\infty}=\inf _{i=1, \ldots, N}\left(\rho^{i}+\sigma \delta^{i}\right)+\frac{\inf _{j=1, \ldots, J} \mu_{j}}{\eta}-\frac{1}{2} \frac{1}{\eta}\left(1+\frac{1}{\eta}\right) \sigma^{2}$.

In order to determine the whole shape of the yield curve, we must determine explicit formulas for the socially efficient discount rates. As seen in Proposition 3.2, we need to analyse how the individual risk tolerances $\tau_{i}$ evolve over time, since they are key features in our analysis. Besides, we have seen that the covariance between individual risk tolerances and individual beliefs and taste parameters play an important part. This means that it is necessary to consider specific settings in order to analyse the shape of the yield curve.

\section{Specific settings and the shape of the yield curve}

We consider the setting with constant parameters. In such a setting, we know that in the standard model with rational beliefs and homogeneous time preference rates, the yield curve is flat and the socially efficient discount rate is given for all $t$ by $R^{t}=\rho+\frac{\mu}{\eta}-\frac{1}{2} \frac{1}{\eta}\left(1+\frac{1}{\eta}\right) \sigma^{2}$. The aim of this section is to analyse the impact of beliefs and time preference rates heterogeneity on the shape of the yield curve.

More precisely, we assume that the aggregate endowment process parameters $\mu$ and $\sigma$ are constant as well as the individual beliefs and time preference parameters $\delta^{i}$ and $\rho^{i}$. We suppose that for all $i$, the relative level of endowment of agent $i$ satisfies $e^{*^{i}}=w_{i} e^{*}$ for some constant $w_{i}$. Moreover, we assume that, for all $i$,

$$
\rho^{i}+\left(\frac{1}{\eta}-1\right)\left(\mu+\sigma \delta^{i}\right)-\frac{1}{2} \frac{1}{\eta}\left(\frac{1}{\eta}-1\right) \sigma^{2}>0
$$

which is a necessary condition for the individual optimization problems to be well defined. Note that assuming constant $\delta^{i}$ s may seem incompatible with learning. However, we consider the case with constant parameters as an approximation of the situation where all the parameters 
are stochastic and where learning is regularly compensated by new shocks on the drift $\mu$.

For all $i$, the individual subjectively anticipated discount rate $R^{t, i}$ is time and state independent and given by $R^{i}=\rho^{i}+\frac{\mu+\sigma \delta^{i}}{\eta}-\frac{1}{2} \frac{1}{\eta}\left(1+\frac{1}{\eta}\right) \sigma^{2}$. We recall that even in this setting the consensus characteristics $\delta_{M}$ and $\rho_{D}$ as well as the aggregation bias $\rho_{B}$ are time-varying, stochastic processes.

\subsection{Logarithmic utility functions}

As we have underlined it in Section 3, the case of logarithmic utility functions is very specific. Indeed, in that case, the socially efficient discount factor can be expressed as a weighted arithmetic average of the individual subjectively anticipated ones, more precisely $A^{t}=\sum_{i} \gamma_{i} A^{t, i}$ (see Proposition 3.1) and there is no aggregation bias $B$ in the expression of the socially efficient discount rate (see Equation 3.3).

Note that Condition (5.1) is equivalent in the logarithmic setting to the condition that all time preference rates be positive. We obtain the following result.

Proposition 5.1. In the case of logarithmic utility agents with positive time preference rates $\rho^{i}$, we have

1. The weight $\gamma_{i}$ of each individual subjectively anticipated discount factor in the socially efficient discount factor is given by $\gamma_{i}=\frac{w_{i} \rho^{i}}{\sum_{j=1}^{N} w_{j} \rho^{j}}, i=1, \ldots, N$.

2. The socially efficient discount rate satisfies

$$
\begin{aligned}
& R^{t}=\mu-\sigma^{2}-\frac{1}{t} \log \left[\sum_{i=1}^{N} \gamma_{i} \exp -\left(\rho^{i}+\delta^{i} \sigma\right) t\right] \text { for all } t>0, \\
& R^{0}=\mu-\sigma^{2}+\sum_{i=1}^{N} \gamma_{i}\left(\rho^{i}+\sigma \delta^{i}\right), \quad R^{\infty}=\mu-\sigma^{2}+\inf _{i=1, \ldots N}\left(\rho^{i}+\sigma \delta^{i}\right) .
\end{aligned}
$$

The yield curve $\left(R^{t}\right)_{t}$ is downward sloping.

We recall that in the standard setting with logarithmic utility functions, the yield curve is flat and for all endowment distribution, the rational discount rate $R^{t}$ is given by $R^{t}=\mu-\sigma^{2}$ for all $t$. When beliefs and time preference rates are heterogeneous, Proposition 5.1 shows that the yield curve (i.e. the socially efficient discount factor as a function of time) is always downward sloping. The behavior of the socially efficient discount factor as a function of beliefs dispersion is more complex since it depends on the correlation between individual characteristics $\left(w_{i}, \rho^{i}, \delta^{i}\right)$.

In order to focus on the impact of beliefs dispersion, Figure 1 and Figure 2 represent the yield curve in a 2 agents setting with no pessimism/optimism on average, i.e. $\delta^{1}+\delta^{2}=0$.

Figure 1 represents the yield curve in the particular setting with $w_{1}=w_{2}$ and $\rho^{1}=\rho^{2}$. In this case, the discount factor is an equally weighted arithmetic average of the individual subjectively anticipated discount factors, as in Weitzman $(1998,2001)$. The short term rate is the rational rate and the long term rate is the pessimistic rate. Moreover, for all $t$, the discount rate $R^{t}$ decreases with beliefs dispersion. 


\section{Insert Figure 1}

The setting with possibly different endowment levels (but still with $\rho^{1}=\rho^{2}$ ) is illustrated in Figure 2. The consensus discount factor is an endowment-weighted arithmetic average of the individual subjective discount factors. The yield curve is still downward sloping. The short term rate is an endowment weighted average of the individually anticipated short term rates, which differs from the rational rate if $w_{1} \neq w_{2}$. If there is a positive correlation between optimism and initial endowment, then the short-term discount rate is higher than in the rational setting and an increase in beliefs heterogeneity increases the short-term rate. The long term rate is still given by the pessimistic rate and an increase in beliefs dispersion always decreases the long term rate. More generally, an increase in beliefs dispersion lowers the yield curve when there is a negative correlation between optimism and initial endowment and rotate it clockwise when there is a positive correlation between optimism and initial endowment. Finally, an increase in the initial relative wealth of the optimistic agent induces a higher short-term discount rate and a greater spread between the short-term discount rate $R^{0}$ and the long-term discount rate $R^{\infty}$, the spread being always positive.

\section{Insert Figure 2}

Table 1 sums up the possible results with two agents who are on average rational, i.e. $\delta^{1}+\delta^{2}=0$. The results are similar with $N$ agents that are on average rational.

If there is a (pessimistic or optimistic) bias on average, i.e. if $\frac{1}{N} \sum_{i=1}^{N} \delta^{i}=\bar{\delta} \neq 0$, then there is an additional optimism (when $\bar{\delta}$ is positive) or pessimism (when $\bar{\delta}$ is negative) effect on the discount rate.

We can consider settings with a continuous distribution on the individual beliefs $\left(\delta^{i}\right)$ as a limit of a setting with a large number of agents. If we suppose that initial endowment is equally distributed ${ }^{20}$, then we obtain as an easy extension of the discrete setting that the discount factor is an average of the individual subjectively anticipated discount factors, i.e. $A^{t}=E^{i}\left[A^{t, i}\right]$. Assuming a Gamma distribution on the individual subjectively anticipated discount rates (resp. a normal distribution on individual beliefs), we retrieve the expression of the discount rate in Weitzman (2001) (resp. in Reinschmidt, 2002).

It is interesting to notice that while the socially efficient discount rate always converges in the long run to the lowest individual subjectively anticipated discount rate, the future short term rates are stochastic and may remain higher than the rational rate and may even converge in the long run to the highest anticipated risk free rate. Consider for instance the setting with $\rho^{1}=\rho^{2}, \delta_{1}+\delta_{2}=0$ and $w_{1}=w_{2}$. It is then easy to obtain that for all $t, \tau_{1}(t)$ and $\tau_{2}(t)$ have the same distribution, which means that none of the agents "wins" and the short term rate remains equal on average to the rational rate. What happens now if, for instance, one agent is pessimistic (or optimistic) with $M^{1}=\mathcal{E}(\delta)$ and the other is rational with $M^{2} \equiv 1$. In this case, the weights $\gamma_{i}$ are the same as in the previous setting since they do not depend upon individual beliefs. However, agent 1 is "wrong" while agent 2 is "right" and it can be shown

\footnotetext{
${ }^{20}$ More precisely, we need that $E^{i}\left[\frac{M^{i}}{\lambda_{i}}\right]=E^{i}\left[M^{i}\right]$.
} 
that $^{21} \tau_{1}(t) \rightarrow_{t \rightarrow \infty} 0$ and $\tau_{2}(t) \rightarrow_{t \rightarrow \infty} 1$, a.s. Hence, by Equation (3.2), the future risk free rate converges in the long run to the rational rate. More generally, when agent 1 is "more wrong" than agent 2 , future short term rates converge to the short term rate that would prevail in the economy made of agent 2 alone, while the socially efficient discount rate converges to the lowest anticipated discount rate.

\subsection{Power utility functions}

We now consider the case of power utility functions. As we have underlined it previously, there are essentially two different settings, $\eta<1$ or $\eta>1$, for which the impact of beliefs dispersion is opposite.

\subsubsection{The case $\eta<1$}

Let us start by considering the specific case $\eta=1 / 2$. In this case, we recall that in the standard setting the yield curve is flat and, for all $t, R^{t}=2 \mu-3 \sigma^{2}$. We consider two agents, who are rational on average. Note that Condition (5.1) is equivalent in this setting to the condition that $\mu-\sigma^{2}+\inf _{i}\left(\rho^{i}+\sigma \delta^{i}\right)>0$.

Proposition 5.2. Consider the case of power utility functions with $\eta=1 / 2$. Suppose that $\delta^{1}=\delta>0, \delta^{2}=-\delta, w_{1}=w_{2}, \rho^{1}=\rho^{2}=0$ and that $\mu-\sigma^{2}-\sigma \delta>0$.

1. The ratio $\left(\frac{\gamma_{1}}{\gamma_{2}}\right)$ is given by

$$
\frac{\gamma_{1}}{\gamma_{2}}=\sqrt{\frac{\mu-\sigma^{2}+\sigma \delta}{\mu-\sigma^{2}-\sigma \delta}}
$$

2. The discount rate is a decreasing function of $t$ and is given by

$$
\begin{aligned}
R^{t} & =2 \mu-3 \sigma^{2}-\frac{1}{t} \ln \left(\left(\gamma_{1}\right)^{2} \exp ^{-2 \delta \sigma t}+\left(\gamma_{2}\right)^{2} \exp ^{2 \delta \sigma t}+2 \gamma_{1} \gamma_{2} \exp ^{-\frac{1}{2} \delta^{2} t}\right) \\
R^{0} & =2 \mu-3 \sigma^{2}+2 \delta \sigma\left(\gamma_{1}-\gamma_{2}\right)-\delta^{2} \gamma_{1} \gamma_{2} \\
R^{\infty} & =2 \mu-3 \sigma^{2}-2 \sigma \delta
\end{aligned}
$$

According to the first point of the proposition, the relative weight of the optimistic agent is greater than the relative weight of the pessimistic agent, i.e., $\gamma_{1}>\gamma_{2}$ : there is an optimistic bias at the aggregate level. This result is valid in the general setting with $\eta<1$ (see Appendix A, Proposition A-4). This implies in particular that an increase in beliefs dispersion leads to an increase of the short term rate. Notice that in the long term an increase in beliefs dispersion leads to a decrease of the socially efficient discount rate since it corresponds to a more pessimistic belief for the more pessimistic agent.

The main result we obtain is the fact that the yield curve is decreasing. We have already seen that the bias between the discount rate $R$ and an average of the $R^{i}$ s is represented (up to a constant) by the beliefs dispersion term $\frac{1}{4} \operatorname{Var}^{\tau}(\delta)$. In the case of logarithmic utility functions, there is no beliefs dispersion term and, as already seen, the socially efficient discount rate $R^{t}$

\footnotetext{
${ }^{21}$ See Yan (2008) for related issues.
} 
decreases with $t$ and converges to the more "pessimistic" individual subjectively anticipated rate. In the case $\eta=\frac{1}{2}$, we have $\frac{\gamma_{2}}{\gamma_{1}}\left(\frac{\tau_{1}}{\tau_{2}}\right)=\left(\frac{M_{t}^{2}}{M_{t}^{1}}\right)^{1 / 2} \sim \ln \mathcal{N}(0, \delta \sqrt{t})$. In particular, this implies that, for large $t$, " $\tau_{1}$ is large with respect to $\tau_{2}$ or $\tau_{2}$ is large with respect to $\tau_{1}$ " with a probability near 1 . Loosely speaking, there are two kinds of states of the world, those for which $\tau_{1}$ vanishes for large $t$ and those for which $\tau_{2}$ vanishes for large $t$. The beliefs dispersion term $\frac{1}{4} \operatorname{Var}^{\tau}(\delta)=\tau_{1} \tau_{2} \delta^{2}$ vanishes then asymptotically. The socially efficient discount rate curve is then globally decreasing and converges, as in the logarithmic case, to the most pessimistic rate. Everything works then as if we had two scenarios one with the optimistic rate and one with the pessimistic rate and the asymptotic socially efficient discount rate is associated to the worst scenario as in Gollier (2002a, b) and Weitzman (1998, 2001). This reasoning is valid for general $\eta<1$. Indeed, we then have $\frac{\gamma_{2}}{\gamma_{1}}\left(\frac{\tau_{1}}{\tau_{2}}\right)=\left(\frac{M_{t}^{2}}{M_{t}^{1}}\right)^{\eta} \sim \ln \mathcal{N}(0,2 \eta \delta \sqrt{t})$, and as in the case $\eta=1 / 2$ the beliefs dispersion term $\frac{1}{2}(1-\eta) \operatorname{Var}^{\tau}(\delta)$ vanishes asymptotically.

In Figure 3 we represent the socially efficient yield curve as well as the rates associated to the $\eta$-average and to the arithmetic average of the individual discount factors ${ }^{22}$. All these curves converge asymptotically to the rate associated to the most pessimistic belief but the " $\eta$ " one is a much better approximation of the yield curve than the "arithmetic" one. The distance between the yield curve and the " $\eta$ " curve measures the impact of the bias due to beliefs dispersion. The variance term increases the short rate but its impact decreases with $t$ and vanishes asymptotically. However this impact can remain non negligible for centuries.

\section{Insert Figure 3}

We have assumed so far that both agents are endowed with the same initial endowment. If we relax this assumption, we still obtain decreasing yield curves converging to the most pessimistic rate. However, when the more optimistic agent has a larger initial endowment, she has a greater weight in the average formula and the impact of her optimism lasts longer. The yield curve has then a higher starting point at $t=0$ and its initial slope is smaller. When the more pessimistic agent has a larger initial endowment, she has a larger weight, the starting point of the yield curve is lower and the convergence to its asymptotic rate is more rapid.

\subsubsection{The case $\eta>1$}

As already underlined, the discount rate exhibits both an average belief/time preference effect that is measured by $\sum \tau_{i} \delta^{i}$ and by $\sum \tau_{i} \rho^{i}$ and a beliefs dispersion effect that is measured by the variance term. As in the case $\eta<1$, the average effect induces a decrease of $R_{t}$ when $t$ increases (since the associated rate converges asymptotically to the lowest rate) and the variance term decreases and vanishes asymptotically. However, in the case $\eta>1$, the beliefs dispersion term $\frac{1}{2}(1-\eta) \operatorname{Var}^{\tau}(\delta)$ is negative. This leads then to two opposite effects when $t$ increases, the average effect inducing a decrease of $R_{t}$ and the dispersion effect inducing an increase of $R_{t}$. Depending on the relative size of these effects, we may obtain decreasing curves as in the case $\eta<1$ as well as increasing then decreasing curves as in Figure 4 . The case $\eta>1$

\footnotetext{
${ }^{22}$ In order to evaluate the integrals and expectations that are involved in the formulas we used the adaptive quadrature implemented in the $\mathrm{R}$ function INTEGRATE to approximate the target integral. This function is based on QUADPACK routines DQAGS and DQAGI (R. Piessens and E. deDoncker-Kapenga,1983) available from Netlib.
} 
leads then to a richer family of possible shapes and is compatible with the fact that long-term rates in bonds markets (i.e. $t=30$ ) are usually higher than short-term rates. In the case of an initially increasing yield curve, this initial shape results from beliefs dispersion and is not retrieved when we approximate the yield curve by the rates associated to the $\eta$-average or the arithmetic average of the individual discount factors as can be seen in Figure 4.

\section{Insert Figure 4}

\section{Conclusion}

When public investment projects entail costs and benefits in the very long run, a question arises about the selection of the relevant discount rate to use for the Costs and Benefits Analysis. Indeed, financial markets do not provide a guideline in this case. In this paper we provide an equilibrium analysis of the effect of expectations heterogeneity and pure time preference rates heterogeneity on the socially efficient discount rate in a (quite general) stochastic setting.

First, we show that the certainty equivalent approach of Weitzman $(1998,2001)$ that consists in taking the (unweighted) arithmetic average of the individually recommended discount factors is compatible with an equilibrium approach if we assume that all utility functions are logarithmic and that all agents have the same initial endowment and the same time preference rate ${ }^{23}$. However, for more general endowment and time preference rate distribution, the average is a weighted average. Furthermore, for more general utility functions, the right concept of average to consider within an equilibrium approach is not the arithmetic average but an $\eta$-average. Finally, there is a bias induced by beliefs and pure time preference rates dispersion.

Beliefs and time preference rate heterogeneity impact the socially efficient discount factor as would an additional source of risk. Increased beliefs and/or time preference rates dispersion leads to lower socially efficient discount rates when $\eta$ is larger than one.

In the long run, we show that the asymptotic socially efficient discount rate is the lowest individual subjectively anticipated discount rate which depends on both distribution of patience and pessimism across agents. This is another element in favor of decreasing discount rates in the long run.

In the short and in the medium term, beliefs heterogeneity leads to a richer class of possible shapes for the yield curve. In particular, we may obtain increasing yield curves for the first 50 years as it is often the case in financial markets.

\section{Appendix}

\section{A Aggregation of individual beliefs and time-preferences}

The aim of this Appendix is to extend the results of Jouini and Napp (2007) to our setting with an infinite horizon and heterogeneous time preference rates. We deal with aggregation issues in the spirit of Varian $(1985,1989)$, Abel (1989), Calvet et al. (2002), Shefrin (2005).

Proposition A-1 We let $N^{i}$ denote the individual composite characteristic $M^{i} D^{i}$.

\footnotetext{
${ }^{23}$ In fact, it suffices that the product of the time preference rate and of the initial endowment be the same for all the agents.
} 
1. The individual characteristics $N^{i}$ can be aggregated into a consensus characteristic $N$ such that

$$
q_{t}^{*}=N_{t} u^{\prime}\left(e_{t}^{*}\right)
$$

with

$$
N=\left[\sum_{i=1}^{N} \gamma_{i}\left(N_{t}^{i}\right)^{\eta}\right]^{1 / \eta}
$$

2. The consensus characteristic $N$ can be written in the form $N=B D M$ where $M$ is a consensus probability belief, $D$ is a pure time consensus discount factor and $B$ is an aggregation bias, related to beliefs dispersion. More precisely, the martingale process $M$ and the finite variation processes $D$ and $B$ satisfy $d M_{t}=\delta_{M} M_{t} d W_{t}, d D_{t}=-\rho_{D} D_{t} d t, d B_{t}=$ $-\rho_{B} B_{t} d t$ with $M_{0}=D_{0}=B_{0}=1$ and

$$
\begin{aligned}
\delta_{M} & =\sum_{i=1}^{N} \tau_{i} \delta^{i}, \quad \rho_{D}=\sum_{i=1}^{N} \tau_{i} \rho^{i} \\
\rho_{B} & =\frac{1}{2}(1-\eta)\left[\sum_{i=1}^{N} \tau_{i}\left(\delta^{i}\right)^{2}-\delta_{M}^{2}\right]=\frac{1}{2}(1-\eta) \operatorname{Var}^{\tau}(\delta)
\end{aligned}
$$

\section{Proof}

1. Since $q^{*}$ is an interior equilibrium price process, we know that there exist Lagrange multipliers $\left(\lambda_{i}\right)$ such that for all $i$ and for all $t$,

$$
\frac{1}{\lambda_{i}} N_{t}^{i} u^{\prime}\left(y_{t}^{i^{*}}\right)=q_{t}^{*}
$$

Since $\sum_{i=1}^{N} y_{t}^{i^{*}}=e_{t}^{*}$, we get

$$
q_{t}^{*}=N_{t} u^{\prime}\left(e_{t}^{*}\right) \text { with } N_{t}=\left[\sum_{i=1}^{N} \gamma_{i}\left(N_{t}^{i}\right)^{\eta}\right]^{1 / \eta} .
$$

2. We can write that $d y_{t}^{i^{*}}=a_{i}(t) d t+b_{i}(t) d W_{t}$ for processes $\left(a_{i}\right)$ and $\left(b_{i}\right)$ such that $\sum_{i=1}^{N} a_{i}(t)=$ $\mu_{t} e_{t}^{*}$ and $\sum_{i=1}^{N} b_{i}(t)=\sigma_{t} e_{t}^{*}$. Analogously, we introduce the processes $\mu_{N}$ and $\delta_{N}$ such that $d N_{t}=\mu_{N}(t) N_{t} d t+\delta_{N}(t) N_{t} d W_{t}$. We apply Itô's Lemma to both sides of Equation (6.2). Identifying the diffusion parts and the drift parts and after simple computations, we obtain

$$
\begin{aligned}
\delta_{N} & =\delta_{M}=\sum_{i=1}^{N} \tau_{i} \delta^{i} \\
\mu_{N} & =\frac{1}{2}(\eta-1)\left[\sum_{i=1}^{N} \tau_{i}\left(\delta^{i}\right)^{2}-\delta_{M}^{2}\right]-\sum_{i=1}^{N} \tau_{i} \rho^{i} .
\end{aligned}
$$

It is easy to check then that $N$ is of the form $N=B D M$. 
Proposition A-2 The risk free rate is given by

$$
r^{f}=\rho_{D}+\frac{1}{\eta}\left[\mu+\delta_{M} \sigma\right]-\frac{1}{2} \frac{1}{\eta}\left(1+\frac{1}{\eta}\right) \sigma^{2}+\rho_{B}
$$

with $\delta_{M}=\sum_{i=1}^{N} \tau_{i} \delta^{i}, \rho_{D}=\sum_{i=1}^{N} \tau_{i} \rho^{i}, \rho_{B}=\frac{1}{2}(1-\eta)\left[\sum_{i=1}^{N} \tau_{i}\left(\delta^{i}\right)^{2}-\delta_{M}^{2}\right] \equiv \frac{1}{2}(1-\eta) \operatorname{Var}^{\tau}(\delta)$. Proof We adopt the notations of Proposition A-1. We let $\mu_{q^{*}}$ (resp. $\sigma_{q^{*}}$ ) denote the drift (resp. diffusion) parameter of the process $q^{*}$, i.e. $d q_{t}^{*}=\mu_{q^{*}} q_{t}^{*} d t+\sigma_{q^{*}} q_{t}^{*} d W_{t}$. Since $q^{*}$ is a state price density, we obtain as in the standard setting that $r^{f}=-\mu_{q^{*}}$. We know from the proof of Proposition A-1 that $q^{*}=N u^{\prime}\left(e^{*}\right)$, hence we get through Itô's Lemma that

$$
\mu_{q^{*}}=\mu_{u^{\prime}\left(e^{*}\right)}+\mu_{N}+\delta_{M}\left(\frac{u^{\prime \prime}\left(e^{*}\right)}{u^{\prime}\left(e^{*}\right)} \sigma e^{*}\right)
$$

where $\mu_{u^{\prime}\left(e^{*}\right)}$ denotes the drift parameter of the process $u^{\prime}\left(e^{*}\right)$. We easily deduce that

$$
r^{f}=\sum_{i=1}^{N} \tau_{i} \rho^{i}+\frac{1}{\eta}\left[\mu+\left(\sum_{i=1}^{N} \tau_{i} \delta^{i}\right) \sigma\right]-\frac{1}{2} \frac{1}{\eta}\left(1+\frac{1}{\eta}\right) \sigma^{2}-\frac{1}{2}(\eta-1) \operatorname{Var}^{\tau}(\delta) .
$$

Proposition A-3 If $\mu$ and $\sigma$ are deterministic and $\delta_{M} \leq 0$, then $-\frac{1}{t} \log E\left[M_{t} u^{\prime}\left(e_{t}^{*}\right)\right] \leq$ $-\frac{1}{t} \log E\left[u^{\prime}\left(e_{t}^{*}\right)\right]$. If we further assume that $\eta>1$ then $-\frac{1}{t} \log E\left[M_{t} B_{t} u^{\prime}\left(e_{t}^{*}\right)\right] \leq-\frac{1}{t} \log E\left[u^{\prime}\left(e_{t}^{*}\right)\right]$. Proof The result can be found in Jouini-Napp (2007) for a finite horizon $T$ and homogeneous time preference rates and is adapted here to the case $T=\infty$ and heterogeneous time preference rates. If $Q_{t}$ is defined on $F_{t}$ by $\frac{d Q_{t}}{d P}=M_{t}$ and letting $W_{s}^{Q_{t}} \equiv W_{s}-\int_{0}^{s} \delta_{M}(u) d u$, we have,

$$
\left(e_{t}^{*}\right)^{-1 / \eta}=\exp \left(-1 / \eta\left[\int_{0}^{t}\left(\mu_{u}-\frac{1}{2} \sigma_{u}^{2}\right) d u+\int_{0}^{t} \sigma_{u} d W_{u}^{Q_{t}}\right]\right) \exp \left(-1 / \eta \int_{0}^{t} \sigma_{u} \delta_{M}(u) d u\right) .
$$

If $\delta_{M} \leq 0$, then $\exp \left(-1 / \eta \int_{0}^{t} \sigma_{u} \delta_{M}(u) d u\right) \geq 1$, hence,

$$
\begin{aligned}
E\left[M_{t}\left(e_{t}^{*}\right)^{-1 / \eta}\right] & \geq E^{Q_{t}}\left[\exp \left(-1 / \eta\left[\int_{0}^{t}\left(\mu_{u}-\frac{1}{2} \sigma_{u}^{2}\right) d u+\int_{0}^{t} \sigma_{u} d W_{u}^{Q_{t}}\right]\right)\right] \\
& \geq E\left[\exp \left(-1 / \eta\left[\int_{0}^{t}\left(\mu_{u}-\frac{1}{2} \sigma_{u}^{2}\right) d u+\int_{0}^{t} \sigma_{u} d W_{u}\right]\right)\right] \\
& \geq E\left[\left(e_{t}^{*}\right)^{-1 / \eta}\right] .
\end{aligned}
$$

It now suffices to remark that $B_{t} \geq 1$, a.s. for $\eta \geq 1$ to conclude.

Proposition A-4 Consider the case of power utility functions. Suppose that $\delta^{1}=\delta>0$ and $\delta^{2}=-\delta, w_{1}=w_{2}$ and $\rho^{1}=\rho^{2}=0$, then there is an optimistic bias $\left(\gamma_{1}>\gamma_{2}\right)$ for $\eta<1$ and a pessimistic bias $\left(\gamma_{1}<\gamma_{2}\right)$ for $\eta>1$.

Proof The result can be found in Jouini-Napp (2007) for a finite horizon $T$ and is adapted here to the case $T=\infty$. Since $w_{1}=w_{2}$, the relative weights $\left(\frac{1}{\lambda_{1}}\right)^{\eta}$ and $\left(\frac{1}{\lambda_{2}}\right)^{\eta}$ must satisfy

$$
\Xi \equiv E\left[\int_{0}^{\infty}\left(e_{t}^{*}\right)^{1-1 / \eta} \frac{\left(M^{1} / \lambda_{1}\right)^{\eta}-\left(M^{2} / \lambda_{2}\right)^{\eta}}{\left\{\left(M^{1} / \lambda_{1}\right)^{\eta}+\left(M^{2} / \lambda_{2}\right)^{\eta}\right\}^{1-1 / \eta}} d t\right]=0 .
$$


It is immediate that $\Xi$ can be written in the form $\Xi=\frac{1}{\lambda_{1}} x^{-1 / \eta} g(x)$ with $x^{2}=\left(\frac{\lambda_{2}}{\lambda_{1}}\right)^{\eta}$ and

$$
g(x)=E\left[\int_{0}^{\infty}\left(e_{t}^{*}\right)^{1-1 / \eta} \frac{x\left(M_{t}^{1}\right)^{\eta}-\frac{1}{x}\left(M_{t}^{2}\right)^{\eta}}{\left\{x\left(M_{t}^{1}\right)^{\eta}+\frac{1}{x}\left(M_{t}^{2}\right)^{\eta}\right\}^{1-1 / \eta}} d t\right] .
$$

For $\eta<1$, we show that $\lambda_{1} \leq \lambda_{2}$. We prove 1) that $g(1) \leq 0$, and 2) that $g$ is increasing with $x$, which implies that for $\lambda_{1}>\lambda_{2}$ we would have $\Xi<0$, which is impossible. We have

$$
g^{\prime}(x)=E\left[\int_{0}^{\infty} \frac{x\left(\left(M_{t}^{1}\right)^{\eta}+\left(M_{t}^{2}\right)^{\eta} / x^{2}\right)^{2}-x\left(\left(M_{t}^{1}\right)^{\eta}-\left(M_{t}^{2}\right)^{\eta} / x^{2}\right)^{2}(1-1 / \eta)}{\left(x\left(M_{t}^{1}\right)^{\eta}+\left(M_{t}^{2}\right)^{\eta} / x\right)^{2-1 / \eta}}\left(e_{t}^{*}\right)^{1-1 / \eta} d t\right],
$$

which is positive for $\eta<1$ and proves 2). Now, $g(1)=\int_{0}^{\infty} E\left[\left(e_{t}^{*}\right)^{1-1 / \eta} \frac{\left(M^{1}\right)^{\eta}-\left(M^{2}\right)^{\eta}}{\left\{\left(M^{1}\right)^{\eta}+\left(M^{2}\right)^{\eta}\right\}^{1-1 / \eta}}\right] d t$. With deterministic coefficients, $\left(M^{1}\right)^{\eta}$ and $\left(M^{2}\right)^{\eta}$ can be written in the form $\left(M^{1}\right)^{\eta}(t)=$ $Z(t)\left(e^{*}(t)\right)^{\frac{\eta \delta}{\sigma}}$ and $\left(M^{2}\right)^{\eta}(t)=Z(t)\left(e^{*}(t)\right)^{-\frac{\eta \delta}{\sigma}}$ for some deterministic process $Z$. It is easy to see that $\frac{\left(M^{1}\right)^{\eta}-\left(M^{2}\right)^{\eta}}{\left\{\left(M^{1}\right)^{\eta}+\left(M^{2}\right)^{\eta}\right\}^{1-1 / \eta}}$ is increasing in $e^{*}$, hence decreasing in $\left(e^{*}\right)^{1-1 / \eta}$, leading to

$$
E\left[\left(e_{t}^{*}\right)^{1-1 / \eta} \frac{\left(M_{t}^{1}\right)^{\eta}-\left(M_{t}^{2}\right)^{\eta}}{\left\{\left(M_{t}^{1}\right)^{\eta}+\left(M_{t}^{2}\right)^{\eta}\right\}^{1-1 / \eta}}\right] \leq E\left[\left(e_{t}^{*}\right)^{1-1 / \eta}\right] E\left[\frac{\left(M_{t}^{1}\right)^{\eta}-\left(M_{t}^{2}\right)^{\eta}}{\left\{\left(M_{t}^{1}\right)^{\eta}+\left(M_{t}^{2}\right)^{\eta}\right\}^{1-1 / \eta}}\right] .
$$

Since $E\left[\frac{\left(M_{t}^{1}\right)^{\eta}-\left(M_{t}^{2}\right)^{\eta}}{\left\{\left(M_{t}^{1}\right)^{\eta}+\left(M_{t}^{2}\right)^{\eta}\right\}^{1-1 / \eta}}\right]=0$, we obtain that $g(1) \leq 0$.

\section{B Proofs}

\section{Proof of Proposition 3.1}

We let $q_{t}^{i^{*}} \equiv M_{t}^{i} D_{t}^{i} u^{\prime}\left(e_{t}^{*}\right)$. By Proposition A-1, we have

$$
q_{t}^{*}=\left[\sum_{i=1}^{N} \gamma_{i}\left(M_{t}^{i} D_{t}^{i}\right)^{\eta}\right]^{1 / \eta} u^{\prime}\left(e_{t}^{*}\right)
$$

This implies that $q^{*}=\left[\sum_{i=1}^{N} \gamma_{i}\left(q_{t}^{i^{*}}\right)^{\eta}\right]^{1 / \eta}$.

1. If $\delta^{i} \equiv \delta$ and if $\rho^{i}(s, \omega) \equiv \rho^{i}(s)$, we have $M^{i} \equiv \widetilde{M}$ for all $i$ and $A^{t, i}=D_{t}^{i} E\left[\widetilde{M}_{t} u^{\prime}\left(e_{t}^{*}\right)\right]$. We 
then have

$$
\begin{aligned}
A^{t} & =E\left[\left(\sum_{i=1}^{N} \gamma_{i}\left(\widetilde{M}_{t} D_{t}^{i} u^{\prime}\left(e_{t}^{*}\right)\right)^{\eta}\right)^{1 / \eta}\right] \\
& =\left(\sum_{i=1}^{N} \gamma_{i}\left(D_{t}^{i}\right)^{\eta}\right)^{1 / \eta} E\left[\widetilde{M}_{t} u^{\prime}\left(e_{t}^{*}\right)\right] \\
& =\left(\sum_{i=1}^{N} \gamma_{i}\left(D_{t}^{i} E\left[\widetilde{M}_{t} u^{\prime}\left(e_{t}^{*}\right)\right]\right)^{\eta}\right)^{1 / \eta} \\
& =\left[\sum_{i=1}^{N} \gamma_{i}\left(A^{t, i}\right)^{\eta}\right]^{1 / \eta} .
\end{aligned}
$$

2. In the case $\eta=1$, we have $A^{t}=E\left[\sum_{i=1}^{N} \gamma_{i} q_{t}^{i^{*}}\right]=\sum_{i=1}^{N} \gamma_{i} E\left[q_{t}^{i^{*}}\right]=\sum_{i=1}^{N} \gamma_{i} A^{t, i}$.

In the case $\eta<1$, we have

$$
\begin{aligned}
\left(A^{t}\right)^{\eta} & =\left\|\sum_{i=1}^{N} \gamma_{i}\left(q_{t}^{i^{*}}\right)^{\eta}\right\|_{1 / \eta} \\
& \leq \sum_{i=1}^{N} \gamma_{i}\left\|\left(q_{t}^{i^{*}}\right)^{\eta}\right\|_{1 / \eta}=\sum_{i=1}^{N} \gamma_{i}\left\|q_{t}^{i^{*}}\right\|_{1}^{\eta}=\sum_{i=1}^{N} \gamma_{i}\left(A^{t, i}\right)^{\eta}
\end{aligned}
$$

hence $A^{t} \leq\left[\sum_{i=1}^{N} \gamma_{i}\left(A^{t, i}\right)^{\eta}\right]^{1 / \eta}$.

In the case $\eta>1$, using Minkovski's Lemma, we get analogously that

$$
E\left\{\left[\sum_{i=1}^{N} \gamma_{i}\left(q_{t}^{i^{*}}\right)^{\eta}\right]^{1 / \eta}\right\}^{\eta} \geq \sum_{i=1}^{N} \gamma_{i} E\left[q_{t}^{i}\right]^{\eta}
$$

hence $A^{t} \geq\left[\sum_{i=1}^{N} \gamma_{i}\left(A^{t, i}\right)^{\eta}\right]^{1 / \eta}$.

When $N^{i} / N^{j}$ is deterministic for all $(i, j)$ we get as in 1 . that $A^{t}=\left[\sum_{i=1}^{N} \gamma_{i}\left(A^{t, i}\right)^{\eta}\right]^{1 / \eta}$.

\section{Proof of Proposition 3.2}

Equation (3.3) results from the definition of $R^{t}=-\frac{1}{t} \log E\left[q_{t}^{*}\right]$ and from Proposition A1. For the second equation, we adopt the notations of Appendix A for the drift anf diffusion parameters of the process $q^{*}$. We then have

$$
\begin{aligned}
& R^{t}=-\frac{1}{t} \log E\left[\exp \int_{0}^{t}\left(\mu_{q^{*}}-\frac{\left(\sigma_{q^{*}}\right)^{2}}{2}\right) d s+\int_{0}^{t} \sigma_{q^{*}} d W_{s}\right]
\end{aligned}
$$

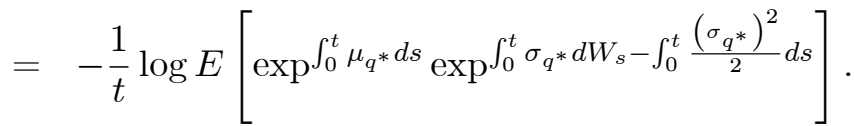


Since $\mu_{q^{*}}=-r^{f}$ (see the proof of Proposition A-2), this implies that

$$
R^{t}=-\frac{1}{t} \log E\left[\exp ^{-\int_{0}^{t} r^{f} d s} \exp ^{\int_{0}^{t} \sigma_{q^{*}} d W_{s}-\int_{0}^{t} \frac{\left(\sigma_{q^{*}}\right)^{2}}{2} d s}\right] .
$$

It is easy to obtain by Proposition A-1 and Itô's Lemma that $\sigma_{q^{*}}=\delta_{M}-\frac{\sigma}{\eta}$. Hence $R^{t}=$ $-\frac{1}{t} \log E^{\bar{Q}_{t}}\left[\exp ^{-\int_{0}^{t} r_{s}^{f} d s}\right]$.

\section{Proof of Proposition 4.1}

We start by identifying bounds for the socially efficient discount rate in 1 . and we prove the result for the asymptotic socially efficient discount rate in 2 .

1. For $\eta=1$, we know by Proposition 3.1 that $A^{t}=\sum_{i=1}^{N} \gamma_{i} A^{t, i}$, hence

$$
\sup _{i}\left(\gamma_{i} A^{t, i}\right) \leq A^{t} \leq \sup _{i} A^{t, i}
$$

For $\eta<1$, we have seen in Proposition 3.1 that $A^{t} \leq\left[\sum_{i=1}^{N} \gamma_{i}\left(A^{t, i}\right)^{\eta}\right]^{1 / \eta}$. Since $\sum_{i=1}^{N} \gamma_{i}=1$, we get that $A^{t} \leq \sup _{i} A^{t, i}$. Moreover, as seen in the proof of Proposition 3.1 we have

$$
A^{t}=E\left[\left(\sum_{i=1}^{N} \gamma_{i}\left(q_{t}^{i^{*}}\right)^{\eta}\right)^{1 / \eta}\right]
$$

hence for all $i$ we have

$$
A^{t} \geq E\left[\left(\gamma_{i}\left(q_{t}^{i^{*}}\right)^{\eta}\right)^{1 / \eta}\right]=E\left[\left(\gamma_{i}\right)^{1 / \eta} q_{t}^{i^{*}}\right]
$$

and

$$
A^{t} \geq \sup _{i}\left(\left(\gamma_{i}\right)^{1 / \eta} A^{t, i}\right)
$$

For $\eta>1$, we get analogously that $A^{t} \geq \sup _{i}\left(\left(\gamma_{i}\right)^{1 / \eta} A^{t, i}\right)$. Moreover, since for all nonnegative real numbers $\left(a_{i}\right)$ we have, for $\eta>1$,

$$
\left(\sum_{i=1}^{N} a_{i}\right)^{1 / \eta} \leq \sum_{i=1}^{N} a_{i}^{1 / \eta}
$$

we then get that, for $\eta>1$,

$$
\begin{aligned}
A^{t} & =E\left[\left(\sum_{i=1}^{N} \gamma_{i}\left(q_{t}^{i^{*}}\right)^{\eta}\right)^{1 / \eta}\right] \\
& \leq E\left[\sum_{i=1}^{N}\left(\gamma_{i}\right)^{1 / \eta} q_{t}^{i^{*}}\right]=\sum_{i=1}^{N}\left(\gamma_{i}\right)^{1 / \eta} A^{t, i} .
\end{aligned}
$$

2. Consider first the case $\eta=1$. Since $A^{t, i}=\exp ^{-R^{t, i} t}$, we have $\sup _{i} A^{t, i}=\exp ^{-\inf _{i} R^{t, i} t}$. Let us denote by $I$ an agent such that $\inf _{i} R^{\infty, i}=R^{\infty, I}$, we have then $\inf _{i} R^{t, i}=R^{t, I}$ for $t$ large enough. Furthermore, it is immediate that $\sup _{i}\left(\gamma_{i} A^{t, i}\right) \geq \gamma_{I} \exp ^{-R^{t, I} t}$. By 1 , we then get, for 
$t$ large enough,

$$
-\frac{1}{t} \log \left(\exp ^{-R^{t, I} t}\right) \leq R^{t} \leq-\frac{1}{t} \log \left(\gamma_{I} \exp ^{-R^{t, I} t}\right)
$$

or equivalently

$$
R^{t, I} \leq R^{t} \leq-\frac{\log \left(\gamma_{i}\right)}{t}+R^{t, I}
$$

hence $\lim _{t \rightarrow \infty} R^{t}=R^{\infty, I}=\inf _{i} R^{\infty, i}$.

Consider the case $\eta<1$. As in the case $\eta=1$ and with the same notations, it is easy to verify that, for $t$ large enough, $\sup _{i}\left(\left(\gamma_{i}\right)^{1 / \eta} A^{t, i}\right) \geq\left(\gamma_{I}\right)^{1 / \eta} \exp ^{-R^{t, I} t}$ and that $\sup _{i} A^{t, i}=\exp ^{-R^{t, I} t}$. Hence, we have $R^{t} \rightarrow_{t \rightarrow \infty} R^{\infty, I}=\inf _{i} R^{\infty, i}$.

Consider now the case $\eta>1$. We have

$$
\sum_{i=1}^{N}\left(\gamma_{i}\right)^{1 / \eta} A^{t, i} \leq\left(\sum_{i=1}^{N}\left(\gamma_{i}\right)^{1 / \eta}\right) \sup _{i} A^{t, i} \leq N \sup _{i} A^{t, i} .
$$

For $t$ large enough, we then have

$$
R^{t, I}-\frac{1}{t} \log N \leq R^{t} \leq R^{t, I}-\frac{1 / \eta}{t} \log \gamma_{I}
$$

and $R^{t} \rightarrow_{t \rightarrow \infty} R^{\infty, I}=\inf _{i} R^{\infty, i}$.

\section{Proof of Proposition 5.1}

1. The relative endowment level $w_{i}$ of agent $i$ must satisfy

$$
w_{i} E\left[\int_{0}^{\infty} q_{t}^{*} e_{t}^{*} d t\right]=E\left[\int_{0}^{\infty} q_{t}^{*} y_{t}^{*^{i}} d t\right]=E\left[\int_{0}^{\infty} \frac{1}{\lambda_{i}} \exp ^{-\rho^{i} t} M_{t}^{i} d t\right]=\frac{1}{\lambda_{i}} \int_{0}^{\infty} \exp ^{-\rho^{i} t} d t=\frac{1}{\lambda_{i} \rho^{i}}
$$

hence $\gamma_{i}=\frac{w_{i} \rho^{i}}{\sum w_{j} \rho^{j}}$.

2. The expression for $R^{t}$ is a direct consequence of the fact that $R^{t}=-\frac{1}{t} \log \left[\sum_{i=1}^{2} \gamma_{i} \exp ^{-R^{i} t}\right]$, obtained in Proposition 3.1. The expression for $R^{0}$ is easily obtained by taking the limit when $t$ converges to 0 in the expression of $R^{t}$. The expression for $R^{\infty}$ results from Proposition 4.1.

\section{Proof of Proposition 5.2}

1. Since $w_{1}=w_{2}$, the relative weights $\gamma_{i}$ must solve

$$
E\left[\int_{0}^{\infty}\left(e_{t}^{*}\right)^{-1}\left[\gamma_{1}\left(M_{t}^{1}\right)^{1 / 2}-\gamma_{2}\left(M_{t}^{2}\right)^{1 / 2}\right]\left[\gamma_{1}\left(M_{t}^{1}\right)^{1 / 2}+\gamma_{2}\left(M_{t}^{2}\right)^{1 / 2}\right] d t\right]=0 .
$$

This is equivalent to

$$
\int_{0}^{\infty} \exp ^{\left(-\mu+\sigma^{2} / 2-\delta^{2} / 2\right) t} E\left[\left(\gamma_{1}\right)^{2} \exp ^{(\delta-\sigma) W_{t}}-\left(\gamma_{2}\right)^{2} \exp ^{(-\delta-\sigma) W_{t}}\right] d t=0
$$

or

$$
\int_{0}^{\infty} \exp \left(-\mu+\sigma^{2} / 2-\delta^{2} / 2\right) t\left(\left(\gamma_{1}\right)^{2} \exp ^{\frac{(\delta-\sigma)^{2}}{2} t}-\left(\gamma_{2}\right)^{2} \exp \frac{\frac{(\delta+\sigma)^{2}}{2} t}{}\right) d t=0
$$

This implies that

$$
\frac{\left(\gamma_{1}\right)^{2}}{\left(\gamma_{2}\right)^{2}}=\frac{\left(-\mu+\sigma^{2}-\sigma \delta\right)}{\left(-\mu+\sigma^{2}+\sigma \delta\right)}
$$


2. We know that $R^{t}=-\frac{1}{t} \log \left[\left\{\sum_{i=1}^{2} \gamma_{i}\left(M_{t}^{i}\right)^{\eta}\right\}^{1 / \eta}\left(e_{t}^{*}\right)^{-1 / \eta}\right]$. It is then easy to obtain that $R^{t}=\frac{\mu}{\eta}-\frac{\sigma^{2}}{2 \eta}+\frac{\delta^{2}}{2}-\frac{1}{t} \ln E\left[\left(\gamma_{1} \exp ^{(\eta \delta-\sigma) W_{t}}+\gamma_{2} \exp ^{(-\eta \delta-\sigma) W_{t}}\right)^{1 / \eta}\right]$. For $\eta=1 / 2$, we have $R^{t}=$ $2 \mu-\sigma^{2}+\frac{\delta^{2}}{2}-\frac{1}{t} \ln V_{t}$ with

$$
V_{t}=E\left[\left(\gamma_{1}\right)^{2} \exp ^{2(\delta / 2-\sigma) W_{t}}+\left(\gamma_{2}\right)^{2} \exp ^{-2(\delta / 2+\sigma) W t}+2 \gamma_{1} \gamma_{2} \exp ^{-2 \sigma W_{t}}\right]
$$

We have then

$$
\begin{aligned}
V_{t} & =\left(\gamma_{1}\right)^{2} \exp ^{2(\delta / 2-\sigma)^{2} t}+\left(\gamma_{2}\right)^{2} \exp ^{2(\delta / 2+\sigma)^{2} t}+2 \gamma_{1} \gamma_{2} \exp ^{2 \sigma^{2} t} \\
& =\exp ^{2 \sigma^{2} t}\left(\left(\gamma_{1}\right)^{2} \exp ^{\left(\delta^{2} / 2-2 \sigma \delta\right) t}+\left(\gamma_{2}\right)^{2} \exp ^{\left(\delta^{2} / 2+2 \sigma \delta\right) t}+2 \gamma_{1} \gamma_{2}\right)
\end{aligned}
$$

hence

$$
R^{t}=2 \mu-3 \sigma^{2}-\frac{1}{t} \ln \left(\left(\gamma_{1}\right)^{2} \exp ^{-2 \delta \sigma t}+\left(\gamma_{2}\right)^{2} \exp ^{2 \delta \sigma t}+2 \gamma_{1} \gamma_{2} \exp ^{-\frac{1}{2} \delta^{2} t}\right) .
$$

The result on $R^{\infty}$ comes from Proposition 4.1. The result on $R^{0}$ comes from taking the limit when $t$ converges to 0 in the expression of $R^{t}$.

\section{References}

[1] Abel, A., 1989. Asset prices under heterogeneous beliefs: implications for the equity premium. Mimeo, University of Pennsylvania.

[2] Blanchard, O. and S. Fischer, 1989. Lectures on Macroeconomics. MIT Press.

[3] Brock, W. and S. Durlauf , 2001. Discrete Choice with Social Interactions. The Review of Economic Studies, 68, 235-260.

[4] Calvet, L., Grandmont, J.-M., and I. Lemaire, 2002. Aggregation of Heterogenous Beliefs and Asset Pricing in Complete Financial Markets. Working Paper.

[5] Caroll, C.D. and M. Kimball, 1996. On the concavity of the consumption function. Econometrica, 64, 981-992.

[6] Chichilnisky, G., 1996, An axiomatic approach to sustainable development. Social Choice and Welfare 13, 231-257.

[7] Cochrane, J., 2001. Asset Pricing. Princeton University Press.

[8] Cox, J., J. Ingersoll and S. Ross, 1985. A theory of the term structure of interest rates. Econometrica, 53, 385-403.

[9] Detemple, J. and S. Murthy, 1994. Intertemporal asset pricing with heterogeneous beliefs. Journal of Economic Theory, 62, 294-320.

[10] Dionne, G. and N. Fombaron, 1996. Non convexities and efficiency of equilibria in insurance markets with asymmetric information. Economics Letters, 52, 31-40. 
[11] Drèze, J.H. and F. Modigliani, 1972. Consumption decisions under uncertainty. Journal of Economic Theory, 5, 308-335.

[12] Dumas, B., Kurshev, A. and R. Uppal, 2008. What can rational investors do about excessive volatility and sentiment fluctuations ? The Journal of Finance. To appear.

[13] Dybvig, P., J. Ingersoll and S. Ross, 1996. Long forward and Zero Coupon rates can never fall. Journal of Business, 69, 1, 1-25.

[14] Gollier, C., 2000. Scientific progress and irreversibility: an economic interpretation of the 'Precautionary Principle'. Journal of Public Economics, 75, 2, 229-253.

[15] Gollier, C., 2002a. Discounting an uncertain future. Journal of Public Economics, 85, 2, 149-166.

[16] Gollier, C., 2002b. Time Horizon and the Discount Rate. Journal of Economic Theory, 107, 463-473.

[17] Gollier, C., 2007. The Consumption-Based Determinants of the Term Structure of Discount Rates. To appear in Mathematics and Financial Economics.

[18] Gollier, C. and M.S. Kimball, 1996. New Methods in the Classical Economics of Uncertainty: Comparing Risks. Papers 96.412, Toulouse - GREMAQ.

[19] Gollier, C. and R. Zeckhauser, 2005. Aggregation of heterogeneous time preferences. Journal of Political Economy, 113, 4, 878-896.

[20] Groom, B., Hepburn, C., Koundouri, P., and D. Pearce, 2005. Declining discount rates: the long and the short of it. Environmental \&Resource Economics, 32, 445-493.

[21] Ingersoll, J.E. and S.A. Ross, 1992. Waiting to invest: investment and uncertainty. Journal of Business, 65, 1-29.

[22] Jouini, E. and C. Napp, 2007. Consensus consumer and intertemporal asset pricing with heterogeneous beliefs. Review of Economic Studies, 74, 1149 - 1174.

[23] Lengwiler, Y., 2005. Heterogeneous patience and the term structure of real interest rates. American Economic Review, 95, 890-896.

[24] Li, C.-Z. and K.-G. Löfgren, 2000. Renewable Resources and Economic Sustainability: A Dynamic Analysis with Heterogeneous Time Preferences. Journal of Environmental Economics and Management, 40, 236-250.

[25] Loewenstein, G. and R. Thaler, 1989. Anomalies. Intertemporal choice. Journal of Economic Perspectives, 3, 181-193.

[26] Loewenstein, G. and D. Prelec, 1992. Anomalies in intertemporal choices: evidence and an interpretation. The Quarterly Journal of Economics. 
[27] Nocetti, D., Jouini, E. and C. Napp, 2008. Properties of the social discount rate in a Benthamite framework with heterogeneous degrees of impatience. To appear, Management Science.

[28] Nordhaus, W., 2007. A Review of the Stern Review of the Economics of Climate Change, Journal of Economic Literature, 45, 686-702.

[29] Piessens, R., deDoncker-Kapenga, E., Uberhuber, C. and D. Kahaner, 1983. Quadpack: a Subroutine Package for Automatic Integration. Springer Verlag.

[30] Reinschmidt, K.F., 2002. Aggregate Social Discount Rate Derived from Individual Discount Rates. Management Science, 48 (2), 307-312.

[31] Shefrin, H., 2005. A Behavioral Approach to Asset Pricing. Elsevier.

[32] Sinclair-Desgagné, B. and H.L. Gabel, 1997. Environmental auditing in management systems and public policy. Journal of Environmental Economics and Management, 33, 331-346.

[33] Varian, H., 1985. Divergence of Opinion in Complete Markets. Journal of Finance, 40, 309-317.

[34] Varian, H., 1989. Difference of Opinion in Financial Markets. In Financial Risk: Theory, Evidence, and Implications. Stone C.C. (Ed.), Kluwer, Dordrecht, The Netherlands.

[35] Vasicek, O., 1977. An equilibrium characterization of the term structure. Journal of Financial Economics, 5, 177-188.

[36] Weitzman, M., 1998. Why the far-distant future should be discounted at its lowest possible rate. Journal of Environmental Economics and Management, 36, 201-208.

[37] Weitzman, M., 2001. Gamma discounting. American Economic Review, 91, 1, 260-271.

[38] Weitzman, M., 2004. Discounting a Distant Future Whose Technology is Unknown. Working Paper.

[39] Weitzman, M., 2007. A Review of The Stern Review of the Economics of Climate Change. Journal of Economic Literature, 45, 703-724.

[40] Yan, H., 2008. Natural Selection in Financial Markets: Does It Work. To appear, Management Science.

[41] Zapatero, F., 1998. Effects of financial innovations on market volatility when beliefs are heterogeneous. Journal of Economic Dynamics and Control, 22, 597-626. 


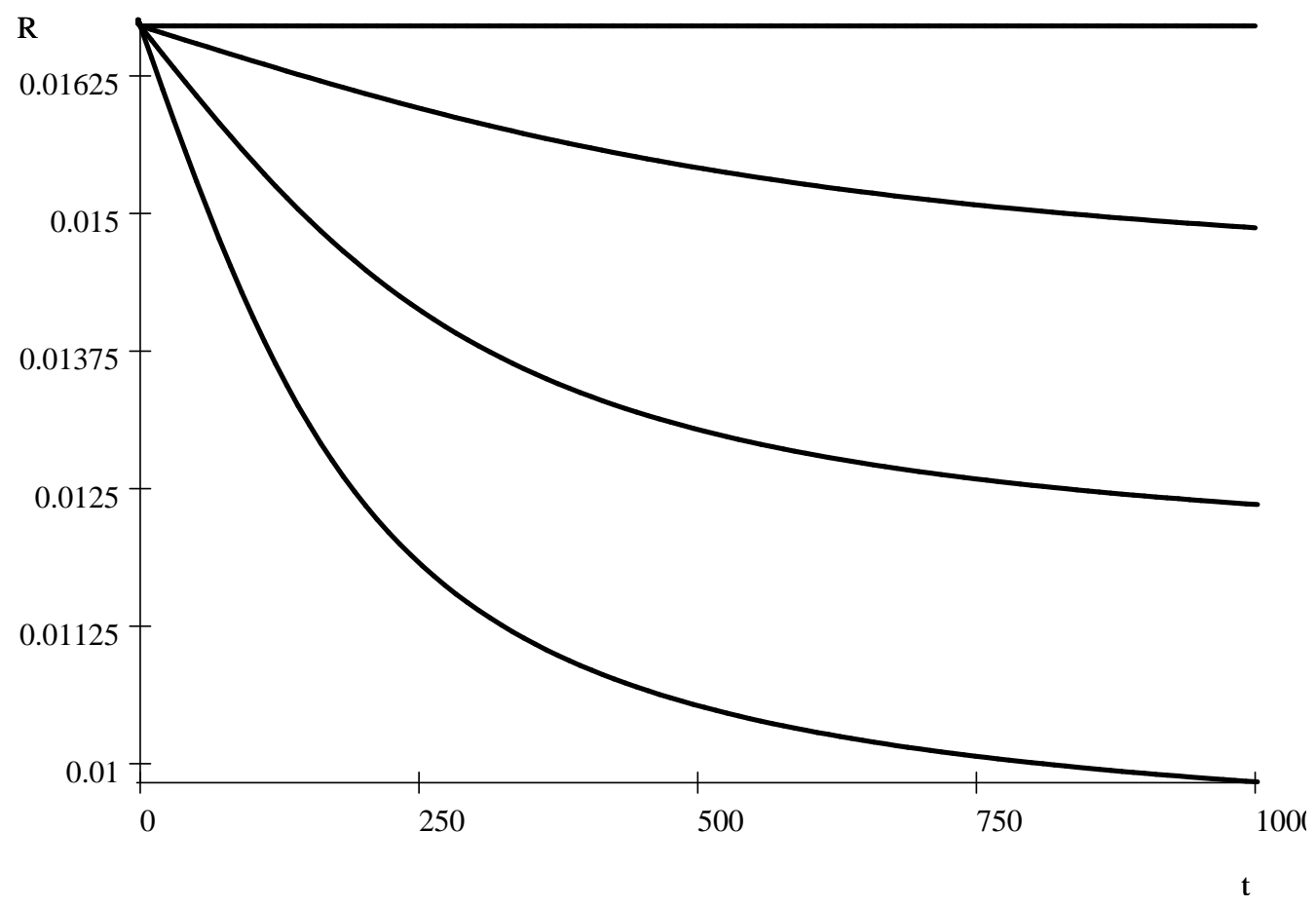

Figure 6.1: This figure represents $R^{t}$ as a function of $t$ in the case of logarithmic utility functions, same time-preference rates $\left(\rho^{1}=\rho^{2}\right)$, same relative initial endowment levels $\left(w_{1}=w_{2}\right)$, and for three different levels of beliefs dispersion $\delta_{j}, j=1,2,3$ (agent 1 has a belief $\delta_{j}$ and agent 2 has a belief $-\delta_{j}$ ). The straight line represents the rational rate. We take $\mu=1.8 \%, \sigma=3.6 \%$ and $\rho$ near 0 . We take $\delta_{1}=0.07, \delta_{2}=0.14, \delta_{3}=0.21$. The three curves are decreasing (see Proposition 5.1). For all $t$, the discount rate $R^{t}$ decreases with the level of beliefs dispersion (see Table 1), hence the three curves do not cross and the lowest curve corresponds to the highest level of beliefs dispersion. The three curves start from the rational rate. Each curve $\mathcal{C}_{j}$ converges asymptotically to the pessimistic discount rate $\rho+\mu-\sigma^{2}-\sigma \delta_{j}$. 


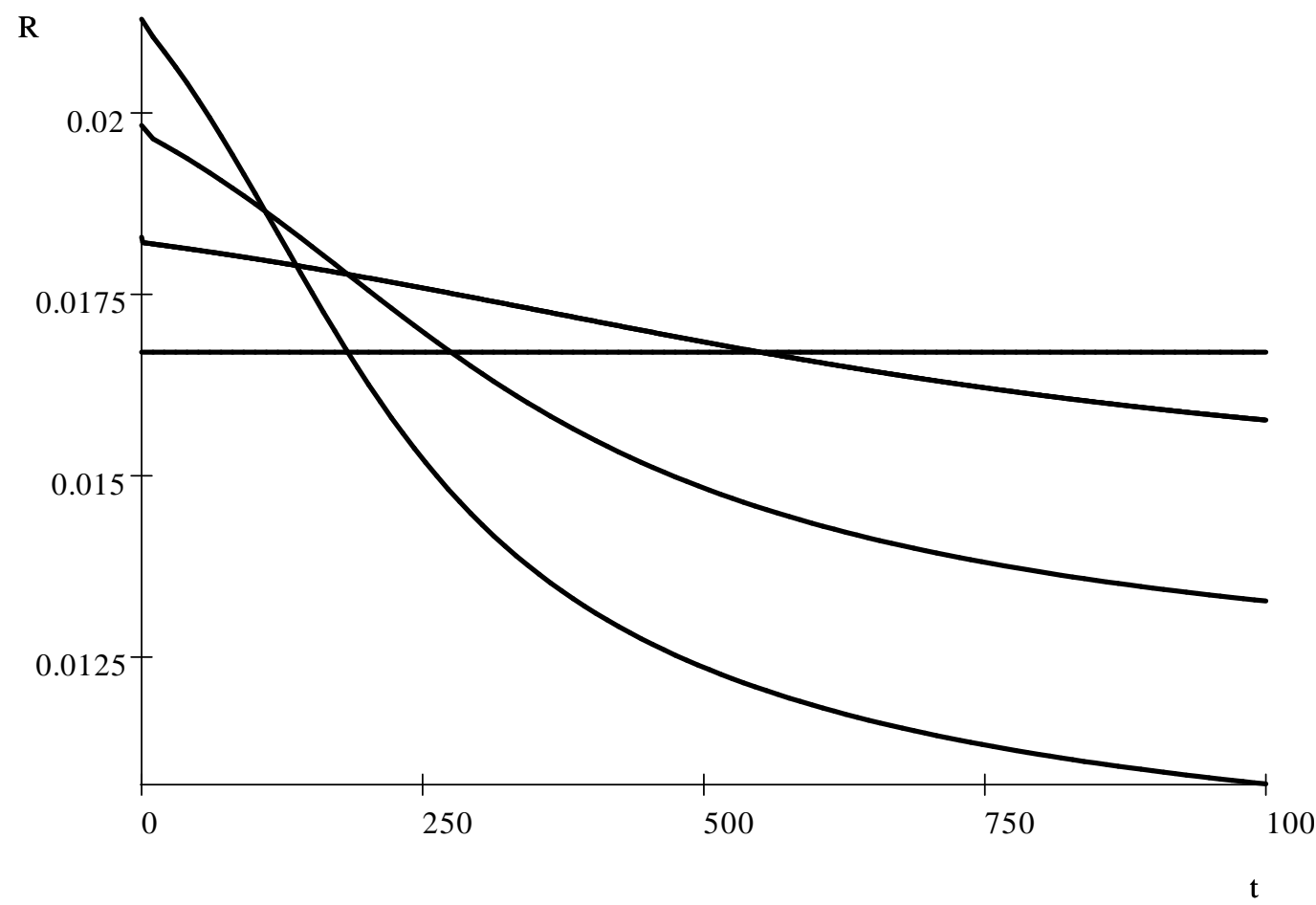

Figure 6.2: This figure represents $R^{t}$ as a function of $t$ for three different levels of beliefs dispersion $\delta_{j}, j=1,2,3$ in the case of logarithmic utility functions, equal time-preference rates $\left(\rho^{1}=\rho^{2}\right)$ and a positive correlation between wealth and optimism. The straight line represents the rational rate. We take $\mu=1.8 \%, \sigma=3.6 \%$ and $\rho$ near $0 ; \delta_{1}=0.07, \delta_{2}=0.14, \delta_{3}=0.21$ (agent 1 has a belief $\delta_{j}$ and agent 2 has a belief $-\delta_{j}$ ); $w_{1}=0.8, w_{2}=0.2$. The three curves are decreasing (Proposition 5.1), each curve $\mathcal{C}_{j}$ converging asymptotically to the pessimistic discount rate $\rho+\mu-\sigma^{2}-\sigma \delta_{j}$. Since $w_{1}>w_{2}$, for small $t$ the discount rate $R^{t}$ increases with the level of beliefs dispersion (Proposition 5.1), and for larger $t$, it decreases with the level of beliefs dispersion, hence the three curves cross. Since $w_{1} \neq w_{2}$, the three curves start from different points, which are given by $\rho+\mu-\sigma^{2}+\left(w_{1}-w_{2}\right) \sigma \delta_{j}$. The lowest curve at $t=1000$ correspond to the highest level of beliefs dispersion. 


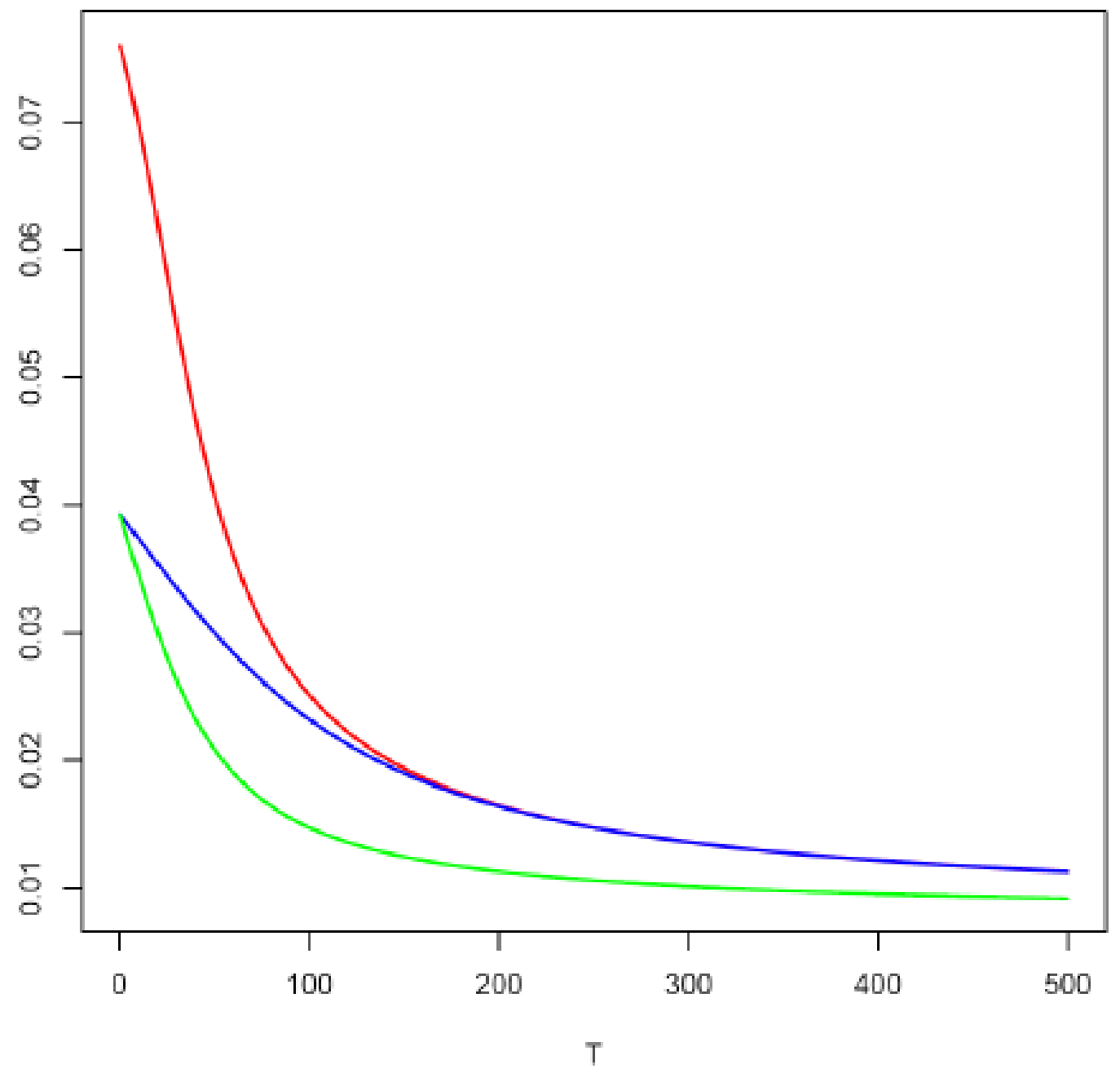

Figure 6.3: This figure represents the socially efficient discount rate for $\gamma_{1}=\gamma_{2}, \eta=0.4, \rho=0$, $\mu=0.018, \sigma=0.036$, and $\delta=0.35$ as well as the curves associated to the $\eta$-average (resp. arithmetic average) of the individual discount factors. The discount rate curve dominates the $\eta$-average that dominates the arithmetic average. With these parameters values, the rational rate is equal to $3.2 \%$. 


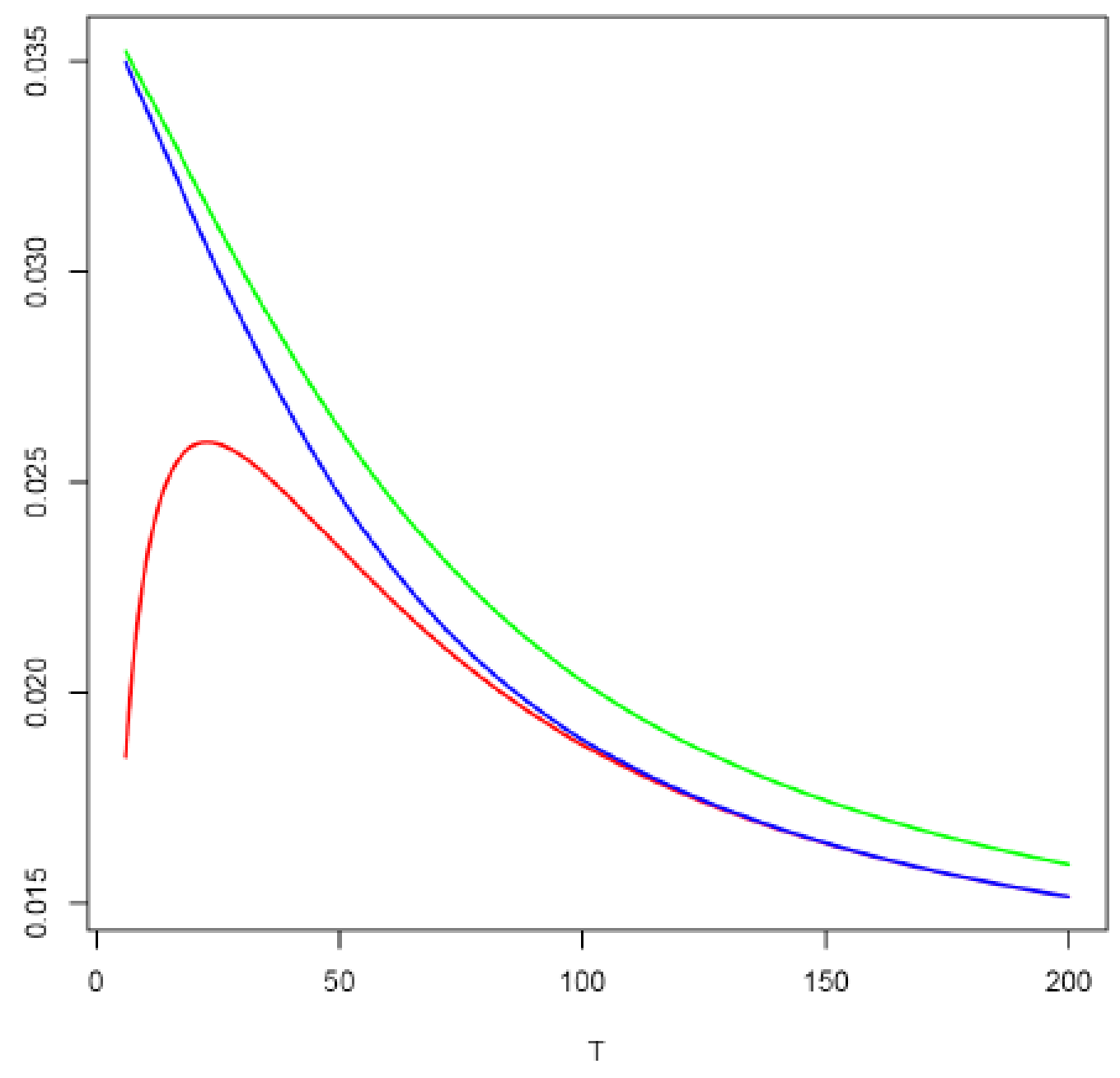

Figure 6.4: This figure represents the socially efficient discount rate for $\gamma_{1}=0.6, \eta=1.2$, $\mu=0.04, \sigma=0.036, \delta=0.7$ as well as the curves associated to the $\eta$-average (resp. arithmetic average) of the individual discount factors. The arithmetic average dominates the $\eta$-average that dominates the discount rate curve. 